\title{
Numerical Methods for Flows Through Porous Media. I
}

\author{
By Michael E. Rose*
}

Abstract. The degenerate parabolic equation

$$
\frac{\partial u}{\partial t}=\nabla \cdot\left(|u|^{\nu} \nabla u\right), \quad \nu \geqslant 1,
$$

has been used to model the flow of gas through a porous medium. Error estimates for continuous and discrete time finite element procedures to approximate the solution of this equation are proved, and several new regularity results are given.

1. A Porous Medium Equation. Introduction. We shall study the porous medium equation

$$
\begin{aligned}
\partial u / \partial t & =\nabla \cdot\left(|u|^{\nu} \nabla u\right) & & \text { on } \Omega \times(0, T], \\
\partial u / \partial n & =0 & & \text { on } \partial \Omega \times[0, T], \\
u(x, 0) & =u_{0}(x) & & \text { on } \Omega,
\end{aligned}
$$

where $\nu \geqslant 1$ is a parameter and $\Omega$ is a bounded domain in $\mathbf{R}^{N}, N \leqslant 3$, with a smooth boundary. The initial function $u_{0}$ is assumed to be nonnegative and four times continuously differentiable on $\bar{\Omega}$. Notice that the compatibility condition $\partial u_{0} / \partial n=0$ holds on $\partial \Omega$.

Our main result is the derivation of error estimates for numerical approximations to the problem (1.1)-(1.3), which we shall refer to as "the porous medium equation" or "PME".

The PME does not, in general, admit classical solutions. Existence and uniqueness of weak solutions was proved in one space dimension by Oleinik, Kalashnikov, and Czou [15], [16] and in several space dimensions by Lions [12]. These proofs concern the PME with different boundary conditions, but the arguments carry over to the PME (1.1)-(1.3).

The maximum principle implies that, since $u_{0}$ is nonnegative on $\Omega, u(x, t)$ is nonnegative for all $(x, t) \in \Omega \times[0, T]$; see [15], [16]. If $u_{0}$ is nonzero, the Neumann boundary condition implies that $u$ will eventually become strictly positive and (1.1) will become nondegenerate for all time $t \geqslant T_{0}, T_{0}$ sufficiently large.

We can rewrite (1.1) in the form

$$
\partial u / \partial t=\Delta K(u) \text { on } \Omega \times(0, T],
$$

Received May 11, 1979; revised July 24, 1981.

1980 Mathematics Subject Classification. Primary 35K65, 65N30, 65N15.

* Current address: Reservoir Engineering Branch, Petroleum Engineering Division, BP Exploration (DOS), Britannic House, Moore Lane, London EC2Y 9BU, United Kingdom. 
where $K(\xi)=\int_{0}^{\xi} k(\tau) d \tau$ and $k(\tau)=|\tau|^{\nu}$. We have defined $k(\tau)$ for the negative reals because, although $u$ is never negative, various numerical approximations to $u$ may take on negative values.

The relations (1.1)-(1.3) represent a model problem for the flow of gas through a porous medium; see [1]. In a sequel to this paper, we have extended our methods to treat a more general porous flow model

$$
\frac{\partial}{\partial t} u+\frac{\partial}{\partial x} f(u)=\frac{\partial}{\partial x}\left(k(u) \frac{\partial u}{\partial x}\right) \quad \text { on } I \times(0, T], I=(0,1) .
$$

In (1.5), $k(u)$ is a nonnegative diffusion coefficient which may vanish for one or more values of $u$. Equation (1.5) has been used to model various problems involving the flow of fluids through porous media, including a one-dimensional waterflood problem in petroleum engineering [8], [17]. The author's treatment of (1.5) has appeared in [18] and [19].

Properties of Solutions of Degenerate Parabolic Equations. The solution of Eq. (1.1) behaves in a strikingly different way than those of nondegenerate parabolic equations (e.g., the heat equation, $\nu=0)$. Let us consider the PME (1.1)-(1.3) as an initial value problem with $\Omega=\mathbf{R}^{1}$.

In 1958, Oleinik, Kalashnikov, and Czou [15], [16] proved that if $u_{0}$ has compact support, then $u(\cdot, t)$ has compact support at any positive time. In fact, it is possible that the support of $u(\cdot, t)$ may not expand at all for $0 \leqslant t \leqslant t_{0}$, for some $t_{0}>0$. The structure of the interface $\partial \operatorname{Supp}(u(x, t))$ has been studied extensively by B. Knerr in his doctoral dissertation [10].

Another distinction between the porous medium equation and nondegenerate parabolic equations is that smooth or real analytic initial data do not necessarily produce a smooth solution. It is well known that nondegenerate parabolic equations possess a 'smoothing' property whereby $L^{2}$ or even distributional initial data yield a smooth solution. Degenerate parabolic equations could be described as having a 'roughing' property.

For $\nu>1$, it has been demonstrated that smooth, compactly supported initial data never yield a $e^{1}$ solution of (1.1) [15], [16]. The space derivative becomes discontinuous at the interface at some positive time.

Oleinik, Kalashnikov, and Czou [16] proved that in one space dimension

$$
\nabla K(u)=|u|^{\nu} \nabla u \in L^{\infty}\left(0, T, L^{\infty}(\Omega)\right) .
$$

In fact, $\nabla K(u)$ is continuous. Aronson [1] has demonstrated that

$$
|u|^{\nu-1} \nabla u \in L^{\propto}\left(0, T, L^{\infty}(\Omega)\right) .
$$

This result is sharp, given his assumptions on the initial data, as shown by the examples cited in Aronson's paper [1]. Further results on the smoothness of $u(x, t)$ and the structure of the interface are contained in [2] and [3].

One can relate these two properties of degenerate parabolic equations. A result of Knerr [10] roughly states that, given smooth initial data with compact support, the support will not expand until $u(x, t)$ becomes nearly vertical at the interface. When the gradient of $u$ becomes discontinuous at the interface, then the support will begin to expand monotonically. 
Outline. The main results of this report are the error estimates we derive for various Galerkin approximations to the solution of (1.1)-(1.3). We begin our analysis in Section 2 by studying several perturbations of (1.1)-(1.3) which yield smooth solutions which approximate the solution of the PME.

In Section 3 we study the regularity theory for (1.1)-(1.3) and a regularized variant of the porous medium equation given by (2.3)-(2.5) below. Theorem 3.3 is a new regularity result for the porous medium equation in a special (but physically important) case which may be of interest aside from its application to deriving error estimates for numerical approximations.

In Section 4 we study error estimates for a Galerkin method to approximate the solution of (1.1)-(1.3). Section 5 contains results for the backward-difference time discretization of the schemes in the previous chapter.

Remarks on Notation. We will use $C$ to denote a positive generic constant. The $L^{2}$ norm and inner product on $\Omega$ shall be given by $\|\cdot\|$ and $(\cdot, \cdot)$ respectively. All other norms and inner products will be labeled by their corresponding function spaces. Finally, if $F$ maps [0,T] into a Banach space $X$, we define the $L^{p}(0, T, X)$ norm by

$$
\|F\|_{L^{p}(0, T, X)}=\left(\int_{0}^{T}\|F\|_{X}^{p} d t\right)^{1 / p} .
$$

This will sometimes be abbreviated to $L^{p}(X)$. For $1 \leqslant p \leqslant \infty$ and a positive integer $m$ we define the spaces

$$
W^{m, p}(\Omega)=\left\{f \in L^{p}(\Omega): \frac{\partial^{\alpha} f}{\partial x^{\alpha}} \in L^{p}(\Omega),|\alpha| \leqslant m\right\}
$$

and the corresponding norms

$$
\|f\|_{W^{m, p}(\Omega)}=\sum_{|\alpha| \leqslant m}\left\|\partial^{\alpha} f / \partial x^{\alpha}\right\|_{L^{p}(\Omega)} .
$$

We use the notation $H^{m}(\Omega)$ to denote $W^{m .2}(\Omega)$. We shall find it convenient to use the norm

$$
\|f\|_{H^{\prime}(\Omega)}=\left\{\|\nabla f\|^{2}+\frac{1}{|\Omega|}\left(\int_{\Omega} f d x\right)^{2}\right\}^{1 / 2}
$$

in place of the (equivalent) $W^{1,2}(\Omega)$ norm.

2. Regularizations of the Porous Medium Equation. One source of difficulty in deriving error estimates for degenerate parabolic problems is the roughness of their solutions. In the special case of a single space dimension or when $\nu=1$ in several dimensions, it is unnecessary to regularize the PME to obtain continuous and discrete time convergence rates. However, when $\nu>1$ in more than one space dimension, we must first perturb the problem (1.1)-(1.3) to obtain a parabolic boundary value problem with a smooth solution $u_{\beta}$. There are several ways to do this.

The method we shall discuss is the technique of nondegenerate parabolic approximation. The diffusion coefficient of $(1.1)$ is

$$
k(\xi)=|\xi|^{\nu}, \quad \nu \geqslant 1 .
$$


We shall replace (2.1) with a new diffusion coefficient

$$
k_{\beta}(\xi) \in e^{4}(\mathbf{R}) \text { for } \beta \in(0,1],
$$

which satisfies the conditions

$$
\begin{array}{ll}
k_{\beta}(\xi)=k(\xi) & \text { for } \xi \geqslant \beta, \\
k_{\beta}(\xi) \geqslant \beta / 2 & \text { for } \xi \geqslant 0, \\
k_{\beta}^{\prime}(\xi) \geqslant 0 & \text { for } \xi \geqslant 0, \text { and } \\
k_{\beta}(-\xi)=k_{\beta}(\xi) . &
\end{array}
$$

Such a regularization could be produced by taking

$$
\operatorname{Max}\left\{\xi, \frac{1}{2} \beta\right\}, \quad \xi \geqslant 0,
$$

rounding off the corner, and extending the result to an even function on the real line. Replacing $k(\xi)$ with $k_{\beta}(\xi)$ yields the nondegenerate parabolic problem

$$
\begin{aligned}
\partial u_{\beta} / \partial t & =\nabla \cdot\left(k_{\beta}\left(u_{\beta}\right) \nabla u_{\beta}\right) & & \text { on } \Omega \times(0, T], \\
\partial u_{\beta} / \partial n & =0 & & \text { on } \partial \Omega \times[0, T], \\
u_{\beta}(x, 0) & =u_{0}(x) & & \text { on } \Omega .
\end{aligned}
$$

Since $k_{\beta}(\xi)$ is in $\mathcal{C}^{4}(\mathbf{R})$ and bounded above zero and $u_{\beta}(x, 0)=u_{0}(x)$ has been chosen so that we have compatibility of the initial and boundary data on $\partial \Omega \times$ $\{t=0\},(2.3)-(2.5)$ is a nondegenerate parabolic problem and $u_{\beta}$ is $e^{4}$ on $\bar{\Omega}$ for all $t>0$ and $e^{2}$ in time [11]. We shall later refer to (2.3)-(2.5) for $\beta=0$; this is the original problem (1.1)-(1.3).

Our next task is to show that $u_{\beta}$ is close to $u$ in an appropriate norm. Towards this end we rewrite the porous medium equation (1.1) in the form

$$
\partial u / \partial t=\Delta K(u)
$$

where

$$
K(\xi)=\int_{0}^{\xi} k(\tau) d \tau=\frac{1}{1+\nu}|\xi|^{\nu} \xi
$$

We also rewrite the nondegenerate equation (2.3) as

$$
\partial u_{\beta} / \partial t=\Delta K_{\beta}\left(u_{\beta}\right)
$$

where

$$
K_{\beta}(\xi)=\int_{0}^{\xi} k_{\beta}(\tau) d \tau
$$

Before estimating $u_{\beta}-u$, we shall need to define an $H^{-1}$ norm on $\Omega$. Let $T$ be the solution operator $w=T f$ of the Neumann problem

$$
\begin{array}{ll}
-\Delta w=f-\bar{f} & \text { on } \Omega, \\
\partial w / \partial n=0 & \text { on } \partial \Omega,
\end{array}
$$

where we define $\bar{f}$ to be the mean value of $f$ on $\Omega$

$$
\bar{f}=\frac{1}{|\Omega|} \int_{\Omega} f d x .
$$


Let

$$
\frac{1}{|\Omega|} \int_{\Omega} w d x=\frac{1}{|\Omega|} \int_{\Omega} f d x=\bar{f}
$$

for uniqueness.

For a function $f(x)$ on $\Omega$ we define the norm $\|f\|_{H^{-1}}$ by

$$
\|f\|_{H^{-1}(\Omega)}=(T f, f)^{1 / 2}=\left\{\|\nabla T f\|^{2}+\frac{1}{|\Omega|}\left(\int_{\Omega} f d x\right)^{2}\right\}^{1 / 2}=\|T f\|_{H^{1}(\Omega)}
$$

THEOREM 2.1. Let $u$ be the solution of (1.1)-(1.3) and let $u_{\beta}$ be the solution of (2.3)-(2.5). Then

$$
\left\|u_{\beta}-u\right\|_{L^{\infty}\left(0, T, H^{-1}(\Omega)\right)}^{2}+\eta\left\|u_{\beta}-u\right\|_{L^{2+\nu}\left(0, T, L^{2+\nu}(\Omega)\right)}^{2+\nu} \leqslant C_{0} \beta^{2+\nu},
$$

where $\eta=\eta(\nu)$ and $C=C_{0}(\nu,|\Omega|)$ are positive constants.

Proof. Using the operator $T$ defined in (2.10)-(2.13), rewrite the equation (2.6) as

$$
T u_{t}+K(u)=\frac{1}{|\Omega|} \int_{\Omega} K(u) d x
$$

at any time $t>0$. Similarly, the regularized $\operatorname{PME~(2.8)~is~equivalent~to~}$

$$
T u_{\beta t}+K_{\beta}\left(u_{\beta}\right)=\frac{1}{|\Omega|} \int_{\Omega} K_{\beta}\left(u_{\beta}\right) d x
$$

for all $t>0$.

We subtract (2.16) from (2.17) to get

$$
\begin{aligned}
& T\left(u_{\beta t}-u_{t}\right)+\left(K\left(u_{\beta}\right)-K(u)\right) \\
& =\left(K\left(u_{\beta}\right)-K_{\beta}\left(u_{\beta}\right)\right)+\frac{1}{|\Omega|} \int_{\Omega}\left(K_{\beta}\left(u_{\beta}\right)-K(u)\right) d x
\end{aligned}
$$

at each positive time. Integrate (2.18) against $u_{\beta}-u$ to get

$$
\begin{aligned}
\left(T\left(u_{\beta t}-u_{t}\right), u_{\beta}-u\right)+ & \left(K\left(u_{\beta}\right)-K(u), u_{\beta}-u\right) \\
= & \left(K\left(u_{\beta}\right)-K_{\beta}\left(u_{\beta}\right), u_{\beta}-u\right) .
\end{aligned}
$$

Notice that, since

$$
\frac{d}{d t} \int_{\Omega}\left(u_{\beta}-u\right) d x=\int_{\Omega}\left(u_{\beta t}-u_{t}\right) d x=0
$$

by the Neumann boundary data (1.2) and (2.4), we have

$$
\begin{aligned}
\int_{\Omega}\left(u_{\beta}(x, t)-u(x, t)\right) d x & =\int_{\Omega}\left(u_{\beta}(x, 0)-u(x, 0)\right) d x \\
& =\int_{\Omega}\left(u_{0}(x)-u_{0}(x)\right) d x=0
\end{aligned}
$$

Thus,

$$
\left(\frac{1}{|\Omega|} \int_{\Omega}\left(K_{\beta}\left(u_{\beta}\right)-K(u)\right) d x, u_{\beta}-u\right)=0
$$


The first term on the left side of (2.19) can be written in the form

$$
\frac{1}{2} \frac{d}{d t}\left\|u_{\beta}-u\right\|_{H^{-1}(\Omega)}^{2} .
$$

To bound the second term on the left side of (2.19) we first use the fact [12], that for any two real numbers $a$ and $b$,

$$
\left(|a|^{\nu} a-|b|^{\nu} b\right) \cdot(a-b) \geqslant \eta|a-b|^{2+\nu}, \quad \eta=\eta(\nu) .
$$

Thus,

$$
\left(K\left(u_{\beta}\right)-K(u), u_{\beta}-u\right) \geqslant \eta\left\|u_{\beta}-u\right\|_{L^{2+\nu}(\Omega)}^{2+\nu}
$$

Consequently,

$$
\frac{1}{2} \frac{d}{d t}\left\|u_{\beta}-u\right\|_{H^{-1}(\Omega)}^{2}+\eta\left\|u_{\beta}-u\right\|_{L^{2+\nu}(\Omega)}^{2+\nu} \leqslant\left(K_{\beta}\left(u_{\beta}\right)-K\left(u_{\beta}\right), u_{\beta}-u\right) .
$$

Use the inequality

$$
a b \leqslant \frac{1}{p} a^{p}+\frac{1}{q} b^{q}, \quad a, b \geqslant 0, \frac{1}{p}+\frac{1}{q}=1
$$

for $p=2+\nu$ and $q=\gamma=(2+\nu) /(1+\nu)$ to bound the right side of (2.23) by

$$
C\left\|K_{\beta}\left(u_{\beta}\right)-K\left(u_{\beta}\right)\right\|_{L^{\gamma}(\Omega)}^{\gamma}+\frac{\eta}{2}\left\|u_{\beta}-u\right\|_{L^{2+\nu}(\Omega)}^{2+\nu}
$$

and hide the second term on the right in (2.25) in the second term on the left in (2.23).

Since $k_{\beta}(\xi)=k(\xi)$ for $\xi \geqslant \beta$, at each $(x, t) \in \Omega \times[0, T]$ we have

$$
\begin{aligned}
\left|K_{\beta}\left(u_{\beta}\right)-K\left(u_{\beta}\right)\right| & =\left|\int_{0}^{\min \left\{u_{\beta}, \beta\right\}}\left(k_{\beta}(\xi)-k(\xi)\right) d \xi\right| \\
& \leqslant \int_{0}^{\beta}\left(\beta^{\nu}-\xi^{\nu}\right) d \xi=\frac{1}{1+\nu} \beta^{1+\nu} .
\end{aligned}
$$

We have used the fact that the maximum principle implies that $u_{\beta}(x, t)$ is nonnegative. Thus,

$$
\begin{gathered}
\left\|K_{\beta}\left(u_{\beta}\right)-K\left(u_{\beta}\right)\right\|_{L^{\gamma}(\Omega)}^{\gamma} \leqslant C \beta^{2+\nu} \\
\frac{1}{2} \frac{d}{d t}\left\|u_{\beta}-u\right\|_{H^{-1}(\Omega)}^{2}+(\eta / 2)\left\|u_{\beta}-u\right\|_{L^{2+\nu}(\Omega)}^{2+\nu} \leqslant C \beta^{2+\nu} .
\end{gathered}
$$

Integrating (2.28) in time from 0 to $T$ establishes the theorem.

There are other ways to regularize the PME (1.1)-(1.3). One regularization which appears in the literature [1], [10], [15], [16] consists of replacing the initial function $u_{0}(x)$ in (1.3) with

$$
u_{0, \alpha}(x)=u_{0}(x)+\alpha
$$

for $0<\alpha \leqslant 1$. Let $u_{\alpha}(x, t)$ denote the solution of (1.1)-(1.2) with the initial function in (2.29). The strong maximum principle for parabolic partial differential equations implies that [11]

$$
u_{\alpha}(x, t) \geqslant \alpha>0 \quad \text { on } \Omega \times[0, T]
$$

so that $k\left(u_{\alpha}\right)$ is bounded above zero and (1.1) becomes nondegenerate. The regularity theory of [11] implies that $u_{\alpha}$ is in the function class $C^{2}\left(0, T, C^{4}(\bar{\Omega})\right)$. 
The argument used to prove Theorem 2.1 can be used to demonstrate that

$$
\left\|u_{\alpha}-u\right\|_{L^{\infty}\left(H^{-1}\right)}^{2}+\eta\left\|u_{\alpha}-u\right\|_{L^{2+\nu}\left(L^{2+\nu}\right)}^{2+\nu} \leqslant C_{0}^{\prime} \alpha^{2} .
$$

The lowered convergence rate is due to the replacement of $(2.26)$ by the bound

$$
\left|K(u)-K\left(u_{\alpha}\right)\right| \leqslant C \alpha \quad \text { on } \Omega \times(0, T] .
$$

This is why we prefer the regularization (2.2)-(2.5).

We shall need a generalization of (2.22) later on: for any integrable functions $f$ and $g$ on $\Omega$ and any $\beta \in[0,1]$,

$$
\eta\|f-g\|_{L^{2+\nu}(\Omega)}^{2+\nu} \leqslant\left(K_{\beta}(f)-K_{\beta}(g), f-g\right),
$$

where the constant $\eta$ is as in (2.22). To verify (2.33), it suffices to show that for all real numbers $a$ and $b$

$$
\eta|a-b|^{2+\nu} \leqslant\left(K_{\beta}(a)-K_{\beta}(b)\right) \cdot(a-b) .
$$

Since $K_{\beta}$ is a monotone increasing function we may assume that $a>b$. By (2.2),

$$
\begin{aligned}
K_{\beta}(a)-K_{\beta}(b) & =\int_{b}^{a} k_{\beta}(\xi) d \xi \geqslant \int_{-(a-b) / 2}^{(a-b) / 2} k_{\beta}(\xi)=2 \int_{0}^{(a-b) / 2} k_{\beta}(\xi) d \xi \\
& \geqslant 2 \int_{0}^{(a-b) / 2} k(\xi) d \xi=\frac{2}{1+\nu}\left(\frac{a-b}{2}\right)^{1+\nu}
\end{aligned}
$$

so that (2.34) holds with $\eta=\left((1+\nu) 2^{\nu}\right)^{-1}$.

3. Regularity Theory. The regularity properties of the solution of the PME (1.1)-(1.3) are not completely understood. When $\operatorname{dim}(\Omega)=1$ or $\nu=1$, it is possible to establish certain $L^{p}$ estimates for $\partial u / \partial t$ which will allow us to prove the highest convergence rate in space that the analysis in Section 4 can produce. When $\operatorname{dim}(\Omega)>1$ and $\nu>1$, our proved spatial convergence rates in the next section are probably not sharp. When $\operatorname{dim}(\Omega)=1$ and $\nu<2$ or when $\nu=1$ in the multidimensional case, the regularity results of this section will yield the highest convergence rates in time that the analysis of Section 5 can produce. When $\nu \geqslant 2$ in the one-dimensional case or $\nu>1$ in several space dimensions, our proved convergence rates in time may not be sharp.

We begin with a collection of basic regularity results.

Lemma 3.1. Let $u_{\beta}$ be the solution of (2.3)-(2.5) for $0 \leqslant \beta \leqslant 1$. Then

$$
\begin{gathered}
\left\|\sqrt{k_{\beta}\left(u_{\beta}\right)} \nabla u_{\beta}\right\|_{L^{2}\left(L^{2}\right)} \leqslant C, \\
\left\|\nabla K_{\beta}\left(u_{\beta}\right)\right\|_{L^{\infty}\left(L^{2}\right)} \leqslant C,
\end{gathered}
$$

and

$$
\left\|K_{\beta}\left(u_{\beta}\right)_{t}\right\|_{L^{2}\left(L^{2}\right)} \leqslant C\left\|\sqrt{k_{\beta}\left(u_{\beta}\right)} u_{\beta t}\right\|_{L^{2}\left(L^{2}\right)} \leqslant C \text {. }
$$

Proof. Integrate (2.3) against $u_{\beta}$ over $\Omega$ to obtain

$$
\frac{1}{2} \frac{d}{d t}\left\|u_{\beta}\right\|^{2}+\left\|\sqrt{k_{\beta}\left(u_{\beta}\right)} \nabla u_{\beta}\right\|^{2}=0
$$


and then integrate in time to prove (3.1). Integrating (2.3) against $K_{\beta}\left(u_{\beta}\right)_{t}$, we see that

$$
\left\|\sqrt{k_{\beta}\left(u_{\beta}\right)} u_{\beta t}\right\|^{2}+\frac{1}{2} \frac{d}{d t}\left\|\nabla K_{\beta}\left(u_{\beta}\right)\right\|^{2}=0 .
$$

Integration in time yields (3.2) and (3.3).

Our next result is based on an $L^{1}$-contraction principle which may be found in Benilan's dissertation [4]. For completeness, we present our own proof.

THEOREM 3.2. Let $u_{\beta}$ be the solution of (2.3)-(2.5) for $0<\beta \leqslant 1$. Then

$$
\left\|\partial u_{\beta} / \partial t\right\|_{L^{x}\left(0, T, L^{\prime}(\Omega)\right)} \leqslant C_{1}
$$

where $C_{1}=\operatorname{Sup}_{0<\beta \leqslant 1}\left\|\Delta K_{\beta}\left(u_{0}\right)\right\|_{I^{\prime}(\Omega)}<\infty$. For the case $\beta=0$, we have

$$
\|\partial u / \partial t\|_{L^{x}(0, T, M(\Omega))} \leqslant C_{1},
$$

where $M(\Omega)$ is the space of finite regular Baire measures on $\Omega$ under the total variation norm.

Proof. Let $u_{\beta}$ and $\tilde{u}_{\beta}$ be two solutions of (2.3)-(2.4) with $0<\beta \leqslant 1$ corresponding to initial data $u_{0}$ and $\tilde{u}_{0}$, respectively. Define the following subsets of $\Omega$

$$
\begin{aligned}
& \Omega_{+}(t)=\left\{x \in \Omega:\left(u_{\beta}-\tilde{u}_{\beta}\right)(x, t)>0\right\}, \\
& \Omega_{-}(t)=\left\{x \in \Omega:\left(u_{\beta}-\tilde{u}_{\beta}\right)(x, t)<0\right\},
\end{aligned}
$$

and notice that

$$
\int_{\Omega_{+}(t)}\left(u_{\beta}-\tilde{u}_{\beta}\right)_{t}(x, t) d x=\int_{\Omega_{+}(t)} \Delta\left(K_{\beta}\left(u_{\beta}\right)-K_{\beta}\left(\tilde{u}_{\beta}\right)\right) d x
$$

We shall prove that

$$
\int_{\Omega_{+}(t)} \Delta\left(K_{\beta}\left(u_{\beta}\right)-K_{\beta}\left(\tilde{u}_{\beta}\right)\right) d x \leqslant 0
$$

for any time $t, 0<t \leqslant T$.

By Sard's Theorem (see Theorem 3.1 of [22]) there is a sequence $\varepsilon_{n} \downarrow 0$ consisting of positive real numbers which are not critical values of $\left(K_{\beta}\left(u_{\beta}\right)-K_{\beta}\left(\tilde{u}_{\beta}\right)\right)(\cdot, t)$ on $\Omega$. Let

$$
\Omega_{+}^{n}(t)=\left\{x \in \Omega:\left(K_{\beta}\left(u_{\beta}\right)-K_{\beta}\left(\tilde{u}_{\beta}\right)\right)(x, t)>\varepsilon_{n}\right\}
$$

for all positive integers $n$. Since $\partial \Omega_{+}^{n}(t)$ is $\mathrm{C}^{1}$, we may use the divergence theorem to obtain

$$
\begin{aligned}
\int_{\Omega_{+}(t)} \Delta\left(K_{\beta}\left(u_{\beta}\right)\right. & \left.-K_{\beta}\left(\tilde{u}_{\beta}\right)\right) d x \\
& =\lim _{n \rightarrow \infty} \int_{\Omega_{+}^{n}(t)} \Delta\left(K_{\beta}\left(u_{\beta}\right)-K_{\beta}\left(\tilde{u}_{\beta}\right)\right) d x \\
& =\lim _{n \rightarrow \infty} \int_{\partial \Omega_{+}^{n}(t)} \frac{\partial}{\partial n}\left(K_{\beta}\left(u_{\beta}\right)-K_{\beta}\left(\tilde{u}_{\beta}\right)\right) d x
\end{aligned}
$$


The integrand of the last term vanishes on $\partial \Omega_{+}^{n}(t) \cap \partial \Omega$ by (2.4). Since $K_{\beta}\left(u_{\beta}\right)-$ $K_{\beta}\left(\tilde{u}_{\beta}\right)$ is greater than $\varepsilon_{n}$ on $\Omega_{+}^{n}(t)$ and equals $\varepsilon_{n}$ on $\partial \Omega_{+}^{n}(t) \cap \Omega$, we have shown that

$$
\frac{\partial}{\partial n}\left(K_{\beta}\left(u_{\beta}\right)-K_{\beta}\left(\tilde{u}_{\beta}\right)\right) \leqslant 0 \quad \text { on } \partial \Omega_{+}^{n}(t) .
$$

Combine (3.8) and (3.9) to establish (3.7).

Bounds (3.6) and (3.7) imply that

$$
\int_{\Omega} \frac{\partial}{\partial t}\left(u_{\beta}-\tilde{u}_{\beta}\right)^{+} d x=\int_{\Omega_{+}(t)} \frac{\partial}{\partial t}\left(u_{\beta}-\tilde{u}_{\beta}\right) d x \leqslant 0 .
$$

Interchanging the roles of $u_{\beta}$ and $\tilde{u}_{\beta}$,

$$
-\int_{\Omega} \frac{\partial}{\partial t}\left(u_{\beta}-\tilde{u}_{\beta}\right)^{-} d x=-\int_{\Omega_{-}(t)} \frac{\partial}{\partial t}\left(u_{\beta}-\tilde{u}_{\beta}\right) d x \leqslant 0 .
$$

Let $\Omega_{0}(t)=\left\{x \in \Omega:\left(u_{\beta}-\tilde{u}_{\beta}\right)(x, t)=0\right\}$; it is easy to see that

$$
\frac{d}{d t} \int_{\Omega_{0}(t)}\left|u_{\beta}-\tilde{u}_{\beta}\right| d x=0 .
$$

These bounds yield the estimate

$$
\begin{aligned}
\frac{d}{d t}\left\|u_{\beta}-\tilde{u}_{\beta}\right\|_{L^{\prime}(\Omega)}= & \frac{d}{d t} \int_{\Omega}\left|u_{\beta}-\tilde{u}_{\beta}\right| d x=\int_{\Omega} \frac{\partial}{\partial t}\left|u_{\beta}-\tilde{u}_{\beta}\right| d x \\
= & \int_{\Omega} \frac{\partial}{\partial t}\left(u_{\beta}-\tilde{u}_{\beta}\right)^{+} d x+\frac{\partial}{\partial t} \int_{\Omega_{0}(t)}\left|u_{\beta}-\tilde{u}_{\beta}\right| d x \\
& -\int_{\Omega} \frac{\partial}{\partial t}\left(u_{\beta}-\tilde{u}_{\beta}\right)^{-} d x \leqslant 0 .
\end{aligned}
$$

Integrate in time to establish the $L^{1}$-contraction result

$$
\left\|\left(u_{\beta}-\tilde{u}_{\beta}\right)(t)\right\|_{L^{\prime}(\Omega)} \leqslant\left\|u_{0}-\tilde{u}_{0}\right\|_{L^{\prime}(\Omega)}
$$

for $0<t \leqslant T$, where we have suppressed the spatial variable.

Let $\tilde{u}_{\beta}(t)=u_{\beta}(t+\Delta t)$ for any positive $\Delta t$, and divide (3.14) by $\Delta t$ to obtain

$$
\left\|\frac{u_{\beta}(t+\Delta t)-u_{\beta}(t)}{\Delta t}\right\|_{L^{\prime}(\Omega)} \leqslant\left\|\frac{u_{\beta}(\Delta t)-u_{\beta}(0)}{\Delta t}\right\|_{L^{\prime}(\Omega)}
$$

for $0<t \leqslant T-\Delta t$. Let $\Delta t \downarrow 0$ to see that

$$
\left\|\partial u_{\beta} / \partial t(t)\right\|_{L^{\prime}(\Omega)} \leqslant\left\|\partial u_{\beta} / \partial t(0)\right\|_{L^{\prime}(\Omega)}=\left\|\Delta K_{\beta}\left(u_{0}\right)\right\|_{L^{\prime}(\Omega)} \leqslant C_{1}
$$

for $0<\beta \leqslant 1$. This proves (3.4) and (3.5) follows immediately from the imbedding of $L^{1}(\Omega)$ into $M(\Omega)$.

In the special case $\nu=1$, a much stronger result can be proved. This case models the isothermal horizontal flow of a perfect gas through a porous medium [17].

TheOREM 3.3. Let $\nu=1$, and let $u$ be the solution of (1.1)-(1.3). Then

$$
\|\partial u / \partial t\|_{L^{\infty}\left(0, T, L^{3}(\Omega)\right)} \leqslant C_{2},
$$

where $C_{2}$ depends on $\operatorname{Min}_{\Omega} \Delta K\left(u_{0}\right),\left\|\nabla K(u)_{t}(x, 0)\right\|$, and $\left\|\Delta K\left(u_{0}\right)\right\|_{L^{3}(\Omega)}$. 
Proof. We begin with the special case where $u_{0}$ is bounded above zero. For any $\alpha>0$, we may replace $u_{0}(x)$ with $u_{0}(x)+\alpha$ to obtain a smooth solution $u_{\alpha}(x, t)$ of (1.1)-(1.3) as in Section 2 satisfying

$$
u_{\alpha}(x, t) \geqslant \alpha>0 \quad \text { on } \Omega \times[0, T] .
$$

We suppress the $\alpha$ subscript until the end of the proof.

Differentiate (1.1) with respect to time, and integrate the result against $K(u)_{t t}$ to obtain

$$
\left(u_{t t}, K(u)_{t t}\right)+\frac{1}{2} \frac{d}{d t}\left\|\nabla K(u)_{t}\right\|^{2}=0
$$

Since $\nu=1$,

$$
K(u)_{t t}=u u_{t t}+u_{t}^{2}
$$

and (3.16) may be rewritten as

$$
\int_{\Omega} u u_{t t}^{2} d x+\frac{1}{3} \frac{d}{d t} \int_{\Omega}\left(u_{t}\right)^{3} d x+\frac{1}{2} \frac{d}{d t}\left\|\nabla K(u)_{t}\right\|^{2}=0 .
$$

Since the first term is nonnegative, integration in time yields

$$
\begin{aligned}
\frac{1}{3} \operatorname{Sup}_{t} \int_{\Omega}\left(u_{t}\right)^{3} d x+ & \frac{1}{2}\left\|\nabla K(u)_{t}\right\|_{L^{x}\left(0, T, L^{2}(\Omega)\right)}^{2} \\
& \leqslant \frac{1}{3} \int_{\Omega} u_{t}(x, 0)^{3} d x+\frac{1}{2}\left\|\nabla K(u)_{t}(x, 0)\right\|^{2} \\
& \leqslant \frac{1}{3}\left\|\Delta K\left(u_{0}\right)\right\|_{L^{3}(\Omega)}^{3}+\frac{1}{2}\left\|\nabla K(u)_{t}(x, 0)\right\|^{2}=C_{2}^{\prime}<\infty
\end{aligned}
$$

We claim that

$$
u_{t}(x, t) \geqslant \operatorname{Min}_{\Omega} \Delta K\left(u_{0}\right)=-C_{2}^{\prime \prime}>-\infty
$$

on $\Omega \times[0, T]$ for a positive constant $C_{2}^{\prime \prime}$. Since $u_{t}(\cdot, t)$ always has mean value zero on $\Omega$, either $u_{t}(\cdot, t)$ is identically zero or it takes on a negative value. Suppose $u_{t}(x, t)$ has a negative minimum at $\left(x_{0}, t_{0}\right)$; we shall verify (3.19) by showing that $t_{0}=0$.

Differentiate (1.1) with respect to time, and let $p=u_{t}$

$$
p_{t}=u \Delta p+2 \nabla u \cdot \nabla p+p \Delta u \quad \text { on } \Omega \times(0, T] .
$$

If $t_{0}>0$, then

$$
0 \geqslant p_{t}=u \Delta p+p \Delta u \geqslant p \Delta u
$$

at $\left(x_{0}, t_{0}\right)$. However, since $p$ is negative and $u$ is positive at $\left(x_{0}, t_{0}\right),(1.1)$ yields

$$
0>p=\Delta K(u)=u \Delta u+(\nabla u)^{2} \geqslant u \Delta u,
$$

and so

$$
\Delta u\left(x_{0}, t_{0}\right)<0 \text {. }
$$

This yields a contradiction in (3.21), and so $p$ must attain its minimum on $\partial \Omega \times(0, T]$ or on $\Omega \times\{t=0\}$. The first possibility is ruled out by the Neumann boundary condition and the strong maximum principle for parabolic partial differential equations. Thus, $t_{0}=0$ and (3.19) is valid. 
Combining (2.24), (3.18), and (3.19), we see that

$$
\begin{aligned}
\int_{\Omega}\left|u_{t}\right|^{3} d x & =\int_{\Omega}\left(u_{t}+2 u_{t}^{-}\right)^{3} d x \\
& =\int_{\Omega}\left\{u_{t}^{3}+6 u_{t}^{2}\left(u_{t}^{-}\right)+12\left(u_{t}^{-}\right)^{2} u_{t}+8\left(u_{t}^{--}\right)^{3}\right\} d x \\
& \leqslant \int_{\Omega} u_{t}^{3} d x+2 \int_{\Omega}\left(u_{t}^{-}\right)^{3} d x \leqslant C_{2}^{\prime}+2|\Omega|^{3}\left(C_{2}^{\prime \prime}\right)^{3}=C_{2}
\end{aligned}
$$

for $0<t \leqslant T$. At the end of the previous section, we saw that $u_{\alpha}(x, t)$ (the solution of (1.1)-(1.3) with $u_{0}(x)$ replaced by $\left.u_{0}(x)+\alpha\right)$ tends to $u(x, t)$ in $L^{\infty}\left(H^{-1}\right)$ and hence distributionally as $\alpha \downarrow 0$. Thus, $\partial u_{\alpha} / \partial t$ converges to $\partial u / \partial t$ distributionally and, since (3.24) yields the bound

$$
\left\|\partial u_{\alpha} / \partial t\right\|_{L^{\infty}\left(0, T, L^{3}(\Omega)\right)} \leqslant C_{2}, \quad 0<\alpha \leqslant 1,
$$

a weak sequential compactness argument allows us to conclude that

$$
\|\partial u / \partial t\|_{L^{\infty}\left(0, T, L^{3}(\Omega)\right)} \leqslant C_{2} .
$$

Estimates (3.18) and (3.19) imply that

$$
\left\|\sqrt{u} u_{t t}\right\|_{L^{2}\left(L^{2}\right)} \leqslant\left(C_{2}^{\prime}\right)^{1 / 2}
$$

and

$$
\left\|\nabla K(u)_{t}\right\|_{L^{\infty}\left(L^{2}\right)} \leqslant\left(C_{2}^{\prime}\right)^{1 / 2}
$$

when $\nu=1$ with $C_{2}^{\prime}$ as above. Also, since $u_{\beta}$ converges to $u$ distributionally, estimates (3.15), (3.25), and (3.26) are valid with $u$ and $K(u)$ replaced by $u_{\beta}$ and $K_{\beta}\left(u_{\beta}\right)$, respectively.

Our next result provides a new proof of an $L^{\infty}$ bound for $\nabla k(u)$ in one space dimension due to D. G. Aronson [1]. We are also able to derive $L^{p}$ bounds, $1 \leqslant p \leqslant \infty$, for $\nabla k(u)$ when $\operatorname{dim}(\Omega)=1$.

LEMMA 3.4. When $\operatorname{dim}(\Omega)=1$ and $1 \leqslant p \leqslant \infty$,

$$
\|\nabla k(u)\|_{L^{\infty}\left(0, T, L^{p}(\Omega)\right)} \leqslant C_{3}=\left\|\nabla k\left(u_{0}\right)\right\|_{L^{p}(\Omega)}
$$

for all $\nu \geqslant 1$.

Proof. As in the proof of the last result, we begin with the solution $u_{\alpha}(x, t)$ of (1.1)-(1.2) with the initial function $u_{0}(x)+\alpha, \alpha>0$, and suppress the subscript until the end of the argument.

For any test function $\phi \in H^{1}(\Omega),(1.1)$ and (1.2) yield

$$
\left(u_{t}, \phi\right)+(k(u) \nabla u, \nabla \phi)=0 .
$$

Choose the following test function

$$
\phi=-k^{\prime}(u) \nabla \cdot\left(|\nabla k(u)|^{p-2} \nabla k(u)\right)
$$


in (3.28), where $p \geqslant 1$. This yields

$$
\begin{aligned}
\left(k(u)_{t},-\nabla \cdot\right. & \left.\left(|\nabla k(u)|^{p-2} \nabla k(u)\right)\right) \\
& +\left(k^{\prime}(u) \nabla \cdot(k(u) \nabla u), \nabla \cdot\left(|\nabla k(u)|^{p-2} \nabla k(u)\right)\right) \\
= & \frac{1}{p} \frac{d}{d t} \int_{\Omega}|\nabla k(u)|^{p} d x \\
& +\left(k(u) k^{\prime}(u) \Delta u+(\nabla k(u))^{2}, \nabla \cdot\left(|\nabla k(u)|^{p-2} \nabla k(u)\right)\right) \\
= & 0 .
\end{aligned}
$$

Use the relation

$$
\begin{aligned}
k(u) \Delta & k(u)=k(u)\left[k^{\prime}(u) \Delta u+k^{\prime \prime}(u)(\nabla u)^{2}\right] \\
& =\left[k(u) k^{\prime}(u) \Delta u+(\nabla k(u))^{2}\right]+\left(k(u) k^{\prime \prime}(u)-\left(k^{\prime}(u)\right)^{2}\right)(\nabla u)^{2} \\
& =\left[k(u) k^{\prime}(u) \Delta u+(\nabla k(u))^{2}\right]-\frac{1}{\nu}(\nabla k(u))^{2}
\end{aligned}
$$

to rewrite (3.30) as

$$
\begin{aligned}
\frac{1}{p} \frac{d}{d t}\|\nabla k(u)\|_{L^{p}(\Omega)}^{p}+\left(k(u) \Delta k(u), \nabla \cdot\left(|\nabla k(u)|^{p-2} \nabla k(u)\right)\right) \\
=-\frac{1}{\nu}\left((\nabla k(u))^{2}, \nabla \cdot\left(|\nabla k(u)|^{p-2} \nabla k(u)\right)\right) .
\end{aligned}
$$

In a single space dimension

$$
\nabla \cdot\left(|\nabla k(u)|^{p-2} \nabla k(u)\right)=(p-1)|\nabla k(u)|^{p-2} \Delta k(u)
$$

and

$$
\begin{aligned}
\int_{\Omega}(\nabla k(u))^{2} & \nabla \cdot\left(|\nabla k(u)|^{p-2} \nabla k(u)\right) d x=(p-1) \int_{\Omega}|\nabla k(u)|^{p} \Delta k(u) d x \\
& =\frac{p-1}{p+1} \int_{\Omega} \nabla \cdot\left(|\nabla k(u)|^{p} \nabla k(u)\right) d x \\
& =\frac{p-1}{p+1} \int_{\partial \Omega}|\nabla k(u)|^{p} \partial k(u) / \partial n d \sigma=0
\end{aligned}
$$

by the Neumann boundary condition (1.2). Thus,

$$
\frac{d}{d t}\|\nabla k(u)\|_{L^{p}(\Omega)} \leqslant 0
$$

for all real numbers $p \geqslant 1$. Consequently

$$
\|\nabla k(u)\|_{L^{\infty}\left(0, T, L^{p}(\Omega)\right)} \leqslant\left\|\nabla k\left(u_{0}\right)\right\|_{L^{p}(\Omega)} .
$$

Letting $p \uparrow \infty$, we obtain

$$
\begin{aligned}
\|\nabla k(u)\|_{L^{\infty}\left(0, T, L^{\infty}(\Omega)\right)} & =\lim _{p \rightarrow \infty}\|\nabla k(u)\|_{L^{\infty}\left(0, T, L^{p}(\Omega)\right)} \\
& \leqslant \lim _{p \rightarrow \infty}\left\|\nabla k\left(u_{0}\right)\right\|_{L^{p}(\Omega)}=\left\|\nabla k\left(u_{0}\right)\right\|_{L^{\infty}(\Omega)} .
\end{aligned}
$$


Since $u_{\alpha}$ converges to $u$ in $L^{2+\nu}\left(L^{2+\nu}\right), k\left(u_{\alpha}\right)$ converges to $k(u)$ almost everywhere and hence in the sense of distributions. It follows that $\nabla k\left(u_{\alpha}\right)$ tends to $\nabla k(u)$ distributionally as $\alpha \downarrow 0$. Since the right side of (3.37) is bounded independent of $\alpha \in(0,1]$, a weak compactness argument implies that

$$
\begin{aligned}
\|\nabla k(u)\|_{L^{\infty}\left(L^{\infty}\right)} & \leqslant \lim _{\alpha \downarrow 0}\left\|\nabla k\left(u_{\alpha}\right)\right\|_{L^{\infty}\left(L^{\infty}\right)} \leqslant \lim _{\alpha \downarrow 0}\left\|\nabla k\left(u_{0}\right)\right\|_{L^{\infty}\left(L^{\infty}\right)} \\
& =\left\|\nabla k\left(u_{0}\right)\right\|_{L^{\infty}(\Omega)} .
\end{aligned}
$$

Estimate (3.27) is valid with $k(u)$ replaced by $k_{\beta}\left(u_{\beta}\right)$. The next lemma is not new but it is unavailable in the literature.

LEMMA 3.5. Let $\nu \geqslant 1$ and let $\mu>\nu / 2-1$. Then

$$
\left\||u|^{\mu} \nabla u\right\|_{L^{2}\left(0, T, L^{2}(\Omega)\right)} \leqslant C_{4},
$$

where $C_{4}$ depends on $\mu, \nu$, and $\left\|u_{0}\right\|_{L^{\infty}(\Omega)}$. In particular, for $\nu<2$,

$$
\|\nabla u\|_{L^{2}\left(0, T, L^{2}(\Omega)\right)} \leqslant C_{4} .
$$

Proof. Once again we begin with the special case where $u_{0}$ is bounded above zero. Since $\|u\|_{L^{\infty}\left(L^{\infty}\right)} \leqslant\left\|u_{0}\right\|_{L^{\infty}\left(L^{\infty}\right)}$, we may assume that $\nu / 2<\mu<\nu / 2-1 / 2$. Let $\phi=J(u)$ in $(3.28)$, where

$$
J(\xi)=\frac{1}{2 \mu-\nu+1} \xi^{2 \mu-\nu+1}, \quad \xi>0
$$

This yields

(3.40) $\left(u_{t}, J(u)\right)+\left(|u|^{\nu} \nabla u, \nabla J(u)\right)=\left(u_{t}, J(u)\right)+\left\||u|^{\mu} \nabla u\right\|_{L^{2}(\Omega)}^{2}=0$.

The first term in (3.40) equals

$$
\begin{aligned}
\frac{d}{d t} \int_{\Omega} \int_{0}^{u} J(\xi) d \xi d x & =\frac{1}{2 \mu-\nu+1} \frac{d}{d t} \int_{\Omega} \int_{0}^{u} \xi^{2 \mu-\nu+1} d \xi d x \\
& =\frac{1}{(2 \mu-\nu+1)(2 \mu-\nu+2)} \frac{d}{d t} \int_{\Omega} u^{2 \mu-\nu+1} d x \\
& =C_{\nu, \mu} \frac{d}{d t} \int_{\Omega} u^{2 \mu-\nu+2} d x
\end{aligned}
$$

Substitute (3.41) into (3.40) and integrate in time to obtain

$$
C_{\nu, \mu} \int_{\Omega}\left\{u(x, T)^{2 \mu-\nu+2}-u(x, 0)^{2 \mu-\nu+2}\right\} d x+\left\||u|^{\mu} \nabla u\right\|_{L^{2}\left(0, T, L^{2}(\Omega)\right)}^{2}=0 .
$$

Thus,

$$
\begin{aligned}
\left\||u|^{\mu} \nabla u\right\|_{L^{2}\left(0, T, L^{2}(\Omega)\right)}^{2} & \leqslant 2|C \nu, \mu|\|u\|_{L^{\infty}\left(L^{\infty}\right)}^{2 \mu-\nu+2} \\
& \leqslant 2\left|C_{\nu, \mu}\right|\left\|u_{0}\right\|_{L^{\infty}\left(L^{\infty}\right)}^{2 \mu-\nu+2}=C_{4}^{2} .
\end{aligned}
$$

Since (3.42) holds for $u_{\alpha}$, all $\alpha>0$, and $\nabla u_{\alpha}$ approaches $\nabla u$ distributionally as $\alpha \downarrow 0$, we see that (3.42) also holds for the solution of (1.1)-(1.3) with nonnegative initial data. 
The last two lemmas may be combined to establish

TheOREM 3.6. Let $\nu<2$ and suppose $\operatorname{dim}(\Omega)=1$. Then

$$
\|\partial u / \partial t\|_{L^{2}\left(0, T, L^{2}(\Omega)\right)} \leqslant C_{5},
$$

where $C_{5}$ depends on $\nu,\left\|u_{0}\right\|_{L^{\infty}(\Omega)}$, and $\left\|\nabla k\left(u_{0}\right)\right\|_{L^{\infty}(\Omega)}$.

Proof. Let $u_{0}$ be bounded above zero at first. Choose $\phi=u_{t}$ in (3.28), and write the result as

$$
\begin{aligned}
\left\|u_{t}\right\|^{2}+\frac{1}{2} & \frac{d}{d t}\left\||u|^{\nu / 2} \nabla u\right\|^{2}=\left(\nu u^{\nu-1} u_{t} \nabla u, \nabla u\right) \\
& =\left(\nabla k(u) u_{t}, \nabla u\right) \leqslant \frac{1}{2}\left\|u_{t}\right\|^{2}+\frac{\nu}{2} \int_{\Omega}|\nabla k(u)|^{2} \cdot(\nabla u)^{2} d x .
\end{aligned}
$$

Hide the first term on the right, and integrate in time; by (3.27) and (3.39)

$$
\begin{aligned}
\left\|u_{t}\right\|_{L^{2}\left(L^{2}\right)}^{2}+ & \left\||u|^{\nu / 2} \nabla u\right\|_{L^{\infty}\left(L^{2}\right)}^{2} \\
& \leqslant\|\nabla k(u)\|_{L^{\infty}\left(L^{\infty}\right)}^{2}\|\nabla u\|_{L^{2}\left(L^{2}\right)}^{2} \leqslant \nu \cdot C_{3}^{2} \cdot C_{4}^{2}=C_{5}^{2} .
\end{aligned}
$$

Our usual weak compactness argument completes the proof.

Estimate (3.43) remains valid if $u_{t}$ is replaced with $u_{\beta t}$. If we use the test function $J(u)_{t}$ in (3.28), where $J(\xi)$ was defined above, then (3.27) and (3.38) may be used to prove that when $\mu>\nu / 2-1$ and $\operatorname{dim}(\Omega)=1$ we have

$$
\left\||u|^{\mu} u_{t}\right\|_{L^{2}\left(0, T, L^{2}(\Omega)\right)} \leqslant C_{6},
$$

where $C_{6}$ depends on $\mu, \nu,\left\|u_{0}\right\|_{L^{\infty}(\Omega)}$, and $\left\|\nabla k\left(u_{0}\right)\right\|_{L^{\infty}(\Omega)}$. As usual, we may replace $u$ by $u_{\beta}$ in (3.46).

Another consequence of the last two lemmas is

TheOREM 3.7. Suppose $\nu<2$ and $\operatorname{dim}(\Omega)=1$. Then

$$
\left\|\nabla K(u)_{t}\right\|_{L^{2}\left(0, T, L^{2}(\Omega)\right)} \leqslant C_{7}
$$

where $C_{7}$ depends on $\nu,\left\|u_{0}\right\|_{L^{\infty}(\Omega)}$, and $\left\|\nabla k\left(u_{0}\right)\right\|_{L^{\infty}(\Omega)}$.

Proof. Suppose $u_{0}$ is bounded above zero, so that $u(x, t)$ is smooth. Differentiate (3.28) with respect to time and choose the test function $\phi=K(u)_{t}$ to obtain

$$
\frac{1}{2} \frac{d}{d t}\left(k(u) u_{t}, u_{t}\right)+\left\|\nabla K(u)_{t}\right\|^{2}=\frac{1}{2}\left(k(u)_{t},\left(u_{t}\right)^{2}\right) .
$$

To bound the right side of (3.48), use $\phi=\frac{1}{2} k(u)_{t} u_{t}$ in (3.28) to see that

$$
\begin{aligned}
\frac{1}{2}\left(k(u)_{t}, u_{t}^{2}\right)= & -\left(\nabla K(u), \nabla\left(\frac{1}{2} k(u)_{t} u_{t}\right)\right) \\
= & -\frac{1}{2}\left(k(u) \nabla u, k^{\prime \prime}(u) \nabla u \cdot u_{t}^{2}\right)-\left(k(u) \nabla u, k^{\prime}(u) u_{t} \nabla u_{t}\right) \\
= & -\frac{1}{2}\left(k(u) \nabla u, k^{\prime \prime}(u) \nabla u \cdot u_{t}^{2}\right)+\left(\nabla k(u) \cdot u_{t}, \nabla k(u) u_{t}\right) \\
& -\left(k(u) \nabla u_{t}+\nabla k(u) \cdot u_{t}, \nabla k(u) \cdot u_{t}\right) \\
= & \left(1-\frac{\nu-1}{2 \nu}\right)\left((\nabla k(u))^{2}, u_{t}^{2}\right)-\left(\nabla k(u)_{t}, \nabla k(u) \cdot u_{t}\right),
\end{aligned}
$$


where we have used the identities

$$
\begin{aligned}
k(u) k^{\prime \prime}(u) & =u^{\nu} \cdot \nu(\nu-1) u^{\nu-2}=\nu(\nu-1) u^{2 \nu-2} \\
& =\frac{\nu-1}{\nu}\left(\nu u^{\nu-1}\right)^{2}=\frac{\nu-1}{\nu}\left(k^{\prime}(u)\right)^{2}
\end{aligned}
$$

and

$$
\nabla\left(k(u) u_{t}\right)=k(u) \nabla u_{t}+\nabla k(u) u_{t} .
$$

Using (3.27), (3.48), (3.49), and the Cauchy-Schwarz inequality to verify the inequality

$$
\text { (3.50) } \begin{aligned}
\frac{1}{2} \frac{d}{d t} \| & \sqrt{k(u)} u_{t}\left\|^{2}+\right\| \nabla K(u)_{t} \|^{2} \\
& \leqslant\left(1-\frac{\nu-1}{2}\right)\|\nabla k(u)\|_{L^{\infty}(\Omega)}^{2}\left\|u_{t}\right\|^{2}+\|\nabla k(u)\|_{L^{\infty}(\Omega)}\left\|\nabla K(u)_{t}\right\|\left\|u_{t}\right\| \\
& \leqslant \frac{1}{2}\left\|\nabla K(u)_{t}\right\|^{2}+C\left\|u_{t}\right\|^{2} .
\end{aligned}
$$

Hide the first term on the right, integrate in time, and use (3.43) to establish

$$
\left\|\sqrt{k(u)} u_{t}\right\|_{L^{\infty}\left(L^{2}\right)}^{2}+\left\|\nabla K(u)_{t}\right\|_{L^{2}\left(L^{2}\right)}^{2} \leqslant C\left\|u_{t}\right\|_{L^{2}\left(L^{2}\right)}^{2} \leqslant C_{7}^{2}
$$

for $\nu<2$. Use a weak compactness argument to complete the proof of (3.47) and the estimate

$$
\left\|\sqrt{K(u)} u_{t}\right\|_{L^{\infty}\left(0, T, L^{2}(\Omega)\right)} \leqslant C_{7}
$$

for $\nu<2$.

Bounds (3.47) and (3.52) remain valid when $u, K$, and $k$ are replaced by $u_{\beta}, K_{\beta}$, and $k_{\beta}$, respectively.

Recently, P. Benilan has demonstrated much stronger $L^{p}$ estimates for $u_{t}$ in one space dimension than we were able to prove in Theorems 3.3 and 3.6 [5].

TheOREM 3.8. Suppose $\operatorname{dim}(\Omega)=1$. Then

$$
\left\|k(u)_{t}\right\|_{L^{\infty}\left(0, T, L^{\infty}(\Omega)\right)} \leqslant C_{8}=C_{8}\left(\nu, u_{0}\right),
$$

for $\nu \geqslant 1$. In particular, when $\operatorname{dim}(\Omega)=1$ and $\nu=1$

$$
\left\|u_{t}\right\|_{L^{\infty}\left(0, T, L^{\infty}(\Omega)\right)} \leqslant C_{8} .
$$

When $\nu>1$, we have

$$
\left\|u_{t}\right\|_{L^{\infty}\left(0, T, L^{q}(\Omega)\right)} \leqslant C_{9}(q, \nu)
$$

for any $q<q^{*}(\nu)=\nu /(\nu-1)$.

We will sometimes use the regularity hypothesis

$$
\left\|u_{t}\right\|_{L^{\gamma}\left(0, T, L^{\gamma}(\Omega)\right)} \leqslant C_{10}=C_{10}(\gamma, \nu),
$$

where $\gamma=(2+\nu) /(1+\nu)<q^{*}(\nu)$ for $\nu \geqslant 1$, in the next two sections. Bound (3.56) is only known to be true in one space dimension (cf. (3.54)-(3.55)) or when $\nu=1$ (cf. (3.15)). Estimates (3.53)-(3.56) are valid when $u$ is replaced by $u_{\beta}, 0<\beta \leqslant 1$. 
4. A Continuous-Time Galerkin Scheme. In this section we shall derive error estimates for a continuous-time Galerkin scheme based on $e^{0}$ piecewise-linear elements. The roughness of the solution of the PME (1.1)-(1.3) implies that no improvement in the asymptotic convergence rates will result from the use of higher-order finite element spaces. Let $\left\{\Delta_{h}\right\}, 0<h \leqslant 1$, be a family of triangulations of $\Omega$; for convenience, we shall assume that the elements $T_{j} \in \Delta_{h}$ cover all of $\Omega$

$$
\Omega=\bigcup_{T_{j} \in \Delta_{h}} T_{j}
$$

Let $\rho\left(T_{j}\right)$ be the radius of the smallest ball containing $T_{j} \in \Delta_{h}$, and let $\sigma\left(T_{j}\right)$ be the radius of the largest ball contained in $T_{j}$. We assume that

$$
h=\operatorname{Max}_{T_{j} \in \Delta_{h}} \rho\left(T_{j}\right)
$$

and that $\left\{\Delta_{h}\right\}$ is a quasiregular family of partitions; i.e. there is a positive constant $L_{0}$ for which

$$
\operatorname{Sup}_{0<h \leqslant 1} \operatorname{Max}_{T_{j} \in \Delta_{h}} \rho\left(T_{j}\right) / \sigma\left(T_{j}\right) \leqslant L_{0} .
$$

We shall frequently make the further assumption that $\left\{\Delta_{h}\right\}$ is a quasiuniform family of triangulations, so that there exists a positive constant $L_{1}$ such that

$$
\operatorname{Sup}_{0<h \leqslant 1} \underset{T_{i}, T_{j} \in \Delta_{h}}{\operatorname{Max}} \rho\left(T_{j}\right) / \rho\left(T_{i}\right) \leqslant L_{1}
$$

holds. We will always indicate when (4.2) is assumed in our results.

Let $\left\{M_{h}\right\}, 0<h \leqslant 1$, be a family of finite-dimensional subspaces of $H^{1}(\Omega)$ defined by

$$
M_{h}=\left\{\chi \in \mathcal{C}^{0}(\bar{\Omega}):\left.\chi\right|_{T_{j}} \text { is linear for each } T_{j} \in \Delta_{h}\right\} .
$$

We shall always use the $H^{1}$ norm given in (1.8) in this and the next section. The quasiregularity hypothesis (4.1) implies the approximation property [7]

$$
\operatorname{Inf}_{\chi \in M_{h}}\|f-\chi\|_{L^{p}(\Omega)} \leqslant C h^{2}\|f\|_{W^{2 . p}(\Omega)}
$$

for all $f$ in $W^{2 . p}(\Omega), 1 \leqslant p \leqslant \infty$. The quasiuniformity hypothesis (4.2) is known to imply the 'inverse' property [7]

$$
\|\chi\|_{H^{\prime}(\Omega)} \leqslant C h^{-1}\|\chi\|, \quad \chi \in M_{h} .
$$

Moreover, (4.3a) implies

$$
\|\chi\| \leqslant C h^{-1}\|\chi\|_{H^{-1}(\Omega)}, \quad \chi \in M_{h},
$$

because

$$
\|\chi\|^{2} \leqslant C\|\chi\|_{H^{-1}(\Omega)}\|\chi\|_{H^{\prime}(\Omega)} \leqslant C h^{-1}\|\chi\|_{H^{-1}(\Omega)}\|\chi\|
$$

for all $\chi$ in $M_{h}$.

Let $\beta$ be a nonnegative parameter, $0 \leqslant \beta \leqslant 1$, and define $H_{\beta}(\xi)=\left(K_{\beta}\right)^{-1}(\xi)$ for real $\xi$, where $K_{\beta}(\xi)$ was defined in (2.9). Our continuous-time Galerkin procedure consists of finding $V_{h}:[0, T] \rightarrow M_{h}$, where $V_{h}$ is the solution of the system of ordinary differential equations

$$
\left(\frac{\partial}{\partial t} H_{\beta}\left(V_{h}\right), \chi\right)+\left(\nabla V_{h}, \nabla \chi\right)=0
$$


for all $\chi$ in $M_{h}$ and $0<t \leqslant T$. We construct our initial function by letting $V_{h}(0) \in M_{h}$ satisfy

$$
P_{h} H_{\beta}\left(V_{h}(0)\right)=P_{h} u_{0},
$$

where $P_{h}$ is the $L^{2}$ projection onto $M_{h}$ given by

$$
\left(P_{h} f, \chi\right)=(f, \chi), \quad \chi \in M_{h},
$$

for $f$ in $L^{2}(\Omega)$.

For $\beta \geqslant 0$, the existence and uniqueness of $V_{h}(0)$ in (4.5) follows from the fact that $P_{h} \circ H_{\beta}$ is a continuous coercive monotone operator on $M_{h}$ and is therefore bijective [6]. The existence and uniqueness of $V_{h}(t)$ for $0<t \leqslant T$ follows from the fundamental theorem of ordinary differential equations.

In (4.4)-(4.5) we have approximated $v_{\beta}=K_{\beta}\left(u_{\beta}\right)$ by $\bigodot^{0}$ piecewise-linear elements instead of approximating $u_{\beta}$ directly. We have done this because we are able to prove a higher convergence rate in the former case. We may then approximate $u_{\beta}$ by $U_{h}=H_{\beta}\left(V_{h}\right)$. For future reference, we rewrite (4.4)-(4.5) as

$$
\left(\frac{\partial}{\partial t} U_{h}, \chi\right)+\left(\nabla K_{\beta}\left(U_{h}\right), \nabla \chi\right)=0
$$

for all $\chi$ in $M_{h}$ and $0<t \leqslant T$, and

$$
P_{h} U_{h}=P_{h} u_{0} .
$$

It is important to note that $U_{h}$ is not piecewise-linear, and hence not an element of $M_{h}$.

We may now state the main results of this section.

Theorem 4.1. Suppose $\operatorname{dim}(\Omega)=1$ and $\nu \geqslant 1$ or that $\operatorname{dim}(\Omega)=2$ or 3 and $\nu=1$. Let $\beta$ be chosen so that $0 \leqslant \beta \leqslant C h^{2 /(1+\nu)}$. Let $V_{h}(t)$ be the solution of (4.4)-(4.5), let $U_{h}=H_{\beta}\left(V_{h}\right)$, and let $u$ be the solution of the PME (1.1)-(1.3). Then

$$
\begin{gathered}
\left\|u-P_{h} U_{h}\right\|_{L^{\infty}\left(H^{-1}\right)} \leqslant C h^{\gamma}, \quad \gamma=\frac{2+\nu}{1+\nu}, \\
\left\|u-U_{h}\right\|_{L^{2+\nu}\left(L^{2+\nu}\right)} \leqslant C h^{2 /(1+\nu)}
\end{gathered}
$$

and

$$
\left\|u-P_{h} U_{h}\right\|_{L^{\infty}\left(L^{2}\right)} \leqslant C h^{1 /(1+\nu)}
$$

Estimates (4.8) and (4.10) require the quasiuniformity assumption (4.2); estimate (4.9) does not.

For $\operatorname{dim}(\Omega)>1$ and $\nu>1$, the known regularity theory for the solution of the PME (1.1)-(1.3) will allow us to demonstrate

THEOREM 4.2. Let $u$ and $U_{h}$ be as above, suppose (4.2) holds, and assume that

$$
\beta=C h^{\sigma}, \quad \sigma=(4+2 \nu) /\left(2+4 \nu+\nu^{2}\right) .
$$

Then

$$
\begin{gathered}
\left\|u-P_{h} U_{h}\right\|_{L^{\infty}\left(H^{-1}\right)} \leqslant C[\ln (1 / h)]^{\alpha /(2+2 \nu)} \cdot h^{((2+\nu) / 2) \sigma}, \\
\left\|u-U_{h}\right\|_{L^{2+\nu}\left(L^{2+\nu}\right)} \leqslant C[\ln (1 / h)]^{\alpha /(1+\nu)(2+\nu)} \cdot h^{\sigma},
\end{gathered}
$$


and

$$
\left\|u-P_{h} U_{h}\right\|_{L^{\infty}\left(L^{2}\right)} \leqslant C[\ln (1 / h)]^{\alpha /(2+2 v)} h^{(2+\nu) / 2 \cdot \sigma-1},
$$

where $\alpha=0$ if $\operatorname{dim}(\Omega)=1$ and $\alpha=1$ if $\operatorname{dim}(\Omega)=2$ or 3 .

The estimates in Theorem 4.2 are probably not sharp. Under certain assumptions on the regularity of the time derivative of the solution of the PME (1.1)-(1.3), we can improve upon the bounds (4.11)-(4.14).

THEOREM 4.3. Suppose the following regularity result is true:

$$
\partial u / \partial t \in L^{\gamma}\left(0, T, L^{\gamma}(\Omega)\right), \quad \gamma=\frac{2+\nu}{1+\nu} .
$$

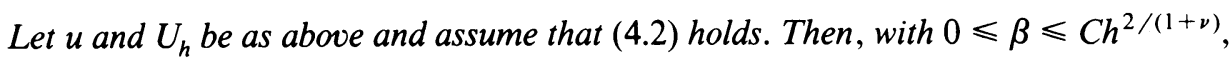

$$
\begin{gathered}
\left\|u-P_{h} U_{h}\right\|_{L^{\infty}\left(H^{-1}\right)} \leqslant C[\ln (1 / h)]^{\alpha /(2+2 v)} h^{\gamma}, \\
\left\|u-U_{h}\right\|_{L^{2+\nu}\left(L^{2+\nu}\right)} \leqslant C[\ln (1 / h)]^{\alpha /(1+\nu)(2+\nu)} \cdot h^{2 /(1+\nu)},
\end{gathered}
$$

and

$$
\left\|u-P_{h} U_{h}\right\|_{L^{\infty}\left(L^{2}\right)} \leqslant C[\ln (1 / h)]^{\alpha /(2+2 v)} h^{1 /(2+\nu)},
$$

where $\alpha=0$ when $\operatorname{dim}(\Omega)=1$ and $\alpha=1$ when $\operatorname{dim}(\Omega)=2$ or 3 .

If we make the stronger regularity hypothesis

$$
\partial u / \partial t \in L^{2}\left(0, T, L^{2}(\Omega)\right),
$$

then estimates (4.16)-(4.18) are valid with $\alpha=0$ for $\operatorname{dim}(\Omega)=1,2$, or 3 .

We begin our analysis by introducing a discrete analogue of the solution operator $T$ defined in (2.10)-(2.13). Let $T_{h}$ be the map from $H^{-1}(\Omega)$ onto $M_{h}$ defined by $W_{h}=T_{h} f$, where

$$
\begin{gathered}
\left(\nabla W_{h}, \nabla \chi\right)=\left(f-\frac{1}{|\Omega|} \int_{\Omega} f d x, \chi\right), \quad \chi \in M_{h}, \\
\int_{\Omega} W_{h} d x=\int_{\Omega} f d x .
\end{gathered}
$$

The restriction of $T_{h}$ to $M_{h}$ is symmetric and positive-definite with respect to the $L^{2}$ inner product. This allows us to define the inner product and norm

$$
\begin{gathered}
(\chi, \psi)_{H_{h}^{-1}}=\left(T_{h} \chi, \psi\right), \quad \chi, \psi \in M_{h}, \\
\|\chi\|_{H_{h}^{-1}}=\left(T_{h} \chi, \chi\right)^{1 / 2}, \quad \chi \in M_{h},
\end{gathered}
$$

on $M_{h}$. Let $f=\psi \in M_{h}$ and $\chi=T_{h} \psi$ in (4.20) to see that

$$
\|\psi\|_{H_{h}^{-1}}=\left\{\left\|\nabla T_{h} \psi\right\|^{2}+\left(\int_{\Omega} \psi d x\right)^{2}\right\}^{1 / 2}=\left\|T_{h} \psi\right\|_{H^{\prime}(\Omega)} .
$$

Since $T_{h}$ is symmetric and positive-semidefinite on $L^{2}(\Omega)$, the $H_{h}^{-1}$ norm on $M_{h}$ extends to a seminorm on all of $H^{-1}(\Omega)$

$$
\|f\|_{H_{h}^{-1}}=\left(T_{h} f, f\right)^{1 / 2}=\left(T_{h} P_{h} f, P_{h} f\right)^{1 / 2}=\left\|P_{h} f\right\|_{H^{-1}(\Omega)}, \quad f \in H^{-1}(\Omega) .
$$


Lemma 4.4. Let the $H^{-1}(\Omega)$ norm be given by (2.14). Then

$$
\|\chi\|_{H_{h}^{-1}} \leqslant\|\chi\|_{H^{-1}(\Omega)}, \quad \chi \in M_{h} .
$$

If we assume that (4.2) holds, then there is a positive constant $\delta$ for which

$$
\delta\|\chi\|_{H^{-1}(\Omega)} \leqslant\|\chi\|_{H_{h}^{-1}}, \quad \chi \in M_{h},
$$

so that the $H_{h}^{-1}$ and $H^{-1}(\Omega)$ norms are equivalent on $M_{h}$.

Proof. Let $E_{h}$ be the projection of $H^{1}(\Omega)$ onto $M_{h}$ given by

$$
\begin{gathered}
\left(\nabla\left(E_{h} f\right), \nabla \chi\right)=(\nabla f, \nabla \chi), \quad \chi \in M_{h}, \\
\int_{\Omega} E_{h} f d x=\int_{\Omega} f d x
\end{gathered}
$$

for $f \in H^{1}(\Omega)$. The definitions of $T$ and $T_{h}$ imply that $T_{h}=E_{h} T$. Use the well-known fact [7] that $\left\|E_{h} f\right\|_{H^{\prime}(\Omega)} \leqslant\|f\|_{H^{\prime}(\Omega)}$ in combination with (2.14) and (4.21) to obtain (4.24)

$$
\begin{aligned}
\|\chi\|_{H_{h}^{-1}} & =\left(T_{h} \chi, \chi\right)^{1 / 2}=\left\|E_{h} T \chi\right\|_{H^{1}(\Omega)} \\
& \leqslant\|T \chi\|_{H^{1}(\Omega)}=(T \chi, \chi)^{1 / 2}=\|\chi\|_{H^{-1}(\Omega)} .
\end{aligned}
$$

By (4.3), we have

$$
\begin{aligned}
\|\chi\| & =\operatorname{Sup}\left\{(\chi, \psi): \psi \in M_{h},\|\psi\| \leqslant 1\right\} \\
& \leqslant \operatorname{Sup}\left\{\|\chi\|_{H_{h}^{-1}}\|\psi\|_{H^{\prime}(\Omega)}: \psi \in M_{h},\|\psi\| \leqslant 1\right\} \\
& \leqslant \operatorname{Sup}\left\{\|\chi\|_{H_{h}^{-1}} C h^{-1}\|\psi\|: \psi \in M_{h},\|\psi\| \leqslant 1\right\} \\
& =C h^{-1}\|\chi\|_{H_{h}^{-1}} .
\end{aligned}
$$

Combine the elliptic regularity result [7]

$$
\|T \phi\|_{H^{2}(\Omega)} \leqslant C\|\phi\|_{L^{2}(\Omega)}, \quad \phi \in H^{2}(\Omega),
$$

with the well-known approximation property of the elliptic projection [7]

$$
\left\|\left(I-E_{h}\right) \psi\right\| \leqslant C h^{2}\|\psi\|_{H^{2}(\Omega)}, \quad \psi \in H^{2}(\Omega),
$$

to see that

$$
\left\|\left(T-T_{h}\right) \phi\right\| \leqslant C h^{2}\|\phi\|, \quad \phi \in L^{2}(\Omega) .
$$

Use (4.21b), (4.28), and (4.31) to obtain (4.25)

$$
\begin{aligned}
\|\chi\|_{H^{-1}(\Omega)}^{2} & =(T \chi, \chi)=\left(E_{h} T \chi, \chi\right)+\left(\left(I-E_{h}\right) T \chi, \chi\right) \\
& =\left(T_{h} \chi, \chi\right)+\left(\left(T-T_{h}\right) \chi, \chi\right) \leqslant\|\chi\|_{H_{h}^{-1}}^{2}+\left(\left(T-T_{h}\right) \chi, \chi\right) \\
& \leqslant\|\chi\|_{H_{h}^{-1}}^{2}+C h^{2}\|\chi\|^{2} \leqslant C\|\chi\|_{H_{h}^{-1}}^{2} .
\end{aligned}
$$

The heart of our argument is contained in the proof of the next result. 
LEMMA 4.5. Let $u_{\beta}(x, t)$ be the solution of (2.1)-(2.3) with $0 \leqslant \beta \leqslant 1$. Let $U_{h}=$ $H_{\beta}\left(V_{h}\right)$, where $V_{h}$ solves (4.4)-(4.5) for the same choice of $\beta$. Then

$$
\begin{aligned}
\left\|u_{\beta}-P_{h} U_{h}\right\|_{L^{\infty}\left(0, T, H_{h}^{-1}\right)}^{2}+ & \int_{0}^{T}\left(K_{\beta}\left(u_{\beta}\right)-K_{\beta}\left(U_{h}\right), u_{\beta}-U_{h}\right) d t \\
& \leqslant C\left\|\left(T-T_{h}\right) \frac{\partial u_{\beta}}{\partial t}\right\|_{L^{\gamma}\left(0, T, L^{\gamma}(\Omega)\right)}^{\gamma},
\end{aligned}
$$

where $\gamma=(2+\nu) /(1+\nu)$.

Proof. Comparing (2.3)-(2.5) for $0 \leqslant \beta \leqslant 1$ with (4.6)-(4.7) yields

$$
\left(\frac{\partial}{\partial t}\left(u_{\beta}-U_{h}\right), \chi\right)+\left(\nabla\left(K_{\beta}\left(u_{\beta}\right)-K_{\beta}\left(U_{h}\right)\right), \nabla \chi\right)=0, \quad \chi \in M_{h},
$$

for $0<t \leqslant T$. Choosing $\chi=1$ in (4.33), we see that

$$
\frac{d}{d t} \int_{\Omega}\left(u_{\beta}-U_{h}\right)(x, t) d x=0, \quad 0 \leqslant t \leqslant T .
$$

By our choice of $U_{h}(0)$ in (4.7), this implies that

$$
\int_{\Omega}\left(u_{\beta}-U_{h}\right)(x, t) d x=\int_{\Omega}\left(I-P_{h}\right) u_{0} d x=0
$$

for $0 \leqslant t \leqslant T$.

Let $\chi=T_{h}\left(u_{\beta}-U_{h}\right)$ in (4.33), and use (4.23), the time-invariance of $T$ and $T_{h}$, the fact that $K_{\beta}\left(U_{h}\right) \in M_{h}$, and (4.34) to obtain

$$
\begin{aligned}
\frac{1}{2} \frac{d}{d t}\left\|u_{\beta}-U_{h}\right\|_{H_{h}^{-1}}^{2}+ & \left(K_{\beta}\left(u_{\beta}\right)-K_{\beta}\left(U_{h}\right), u_{\beta}-U_{h}\right) \\
= & -\left(\left(I-E_{h}\right) K_{\beta}\left(u_{\beta}\right), u_{\beta}-U_{h}\right) .
\end{aligned}
$$

Use (2.17) to rewrite the right side of (4.35) as

$$
\left(\left(T-T_{h}\right) \partial u_{\beta} / \partial t, u_{\beta}-U_{h}\right)
$$

and use Hölder's inequality, (2.24), and (2.33) to bound it by

$$
\begin{aligned}
& \left\|\left(T-T_{h}\right) \partial u_{\beta} / \partial t\right\|_{L^{\gamma}(\Omega)}\left\|u_{\beta}-U_{h}\right\|_{L^{2+\nu}(\Omega)} \\
& \quad \leqslant C\left\|\left(T-T_{h}\right) \partial u_{\beta} / \partial t\right\|_{L^{\gamma}(\Omega)}^{\gamma}+\frac{\eta}{2}\left\|u_{\beta}-U_{h}\right\|_{L^{2+\nu}(\Omega)}^{2+\nu} \\
& \quad \leqslant C\left\|\left(T-T_{h}\right) \partial u_{\beta} / \partial t\right\|_{L^{\gamma}(\Omega)}^{\gamma}+\frac{1}{2}\left(K_{\beta}\left(u_{\beta}\right)-K_{\beta}\left(U_{h}\right), u_{\beta}-U_{h}\right) .
\end{aligned}
$$

Absorb the last term in the second term on the left side of (4.35), and integrate in time to obtain (4.32).

LEMMA 4.6. Let $P_{h}$ be the orthogonal projection of $L^{2}(\Omega)$ onto $M_{h}$ with respect to the $L^{2}$ inner product, and let $u_{\beta}$ be the solution of (2.3)-(2.5), $0 \leqslant \beta \leqslant 1$. Then

$$
\left\|\left(I-P_{h}\right) u_{\beta}\right\|_{L^{\infty}\left(L^{2}\right)} \leqslant C h^{1 /(1+\nu)}
$$

and

$$
\left\|\left(I-P_{h}\right) u_{\beta}\right\|_{L^{\infty}\left(H^{-1}\right)} \leqslant C h^{\gamma} .
$$


Proof. Let $u_{\beta}^{\varepsilon}$ be defined by

$$
u_{\beta}^{\varepsilon}(x, t)=\operatorname{Max}\left\{u_{\beta}(x, t), \varepsilon\right\} \quad \text { on } \Omega \times[0, T]
$$

for any $\varepsilon>0$. Since $k_{\beta}\left(u_{\beta}\right)(x, t) \geqslant \frac{1}{2} \varepsilon$ whenever $u_{\beta}(x, t) \geqslant \varepsilon$ by (2.2), bound (3.2) implies

$$
\left\|\nabla u_{\beta}^{\varepsilon}\right\|_{L^{\infty}\left(0, T, L^{2}(\Omega)\right)} \leqslant C \varepsilon^{-\nu} .
$$

Use the approximation property of the $L^{2}$ projection [7]

$$
\left\|\left(I-P_{h}\right) \phi\right\|_{H^{j}(\Omega)} \leqslant C h\|\phi\|_{H^{j+1}(\Omega)}
$$

for $\phi \in H^{j+1}(\Omega), j=-1$ or 0 , together with (4.39) and the boundedness of $P_{h}$ as an operator on $L^{2}(\Omega)$ to see that

$$
\begin{aligned}
\|\left(I-P_{h}\right) u_{\beta} & \|_{L^{\infty}\left(0, T, L^{2}(\Omega)\right)} \\
& \leqslant\left\|\left(I-P_{h}\right)\left(u_{\beta}-u_{\beta}^{\varepsilon}\right)\right\|_{L^{\infty}\left(0, T, L^{2}(\Omega)\right)}+\left\|\left(I-P_{h}\right) u_{\beta}^{\varepsilon}\right\|_{L^{\infty}\left(0, T, L^{2}(\Omega)\right)} \\
& \leqslant C\left\|u_{\beta}^{\varepsilon}-u_{\beta}\right\|_{L^{\infty}\left(0, T, L^{2}(\Omega)\right)}+C h\left\|\nabla u_{\beta}^{\varepsilon}\right\|_{L^{\infty}\left(0, T, L^{2}(\Omega)\right)} \\
& \leqslant C\left(\varepsilon+h \varepsilon^{-\nu}\right)
\end{aligned}
$$

Letting $\varepsilon=h^{1 /(1+\nu)}$, we see that (4.41) implies (4.37). Next, use (4.40) with $j=1$, bound (4.41) and the idempotence of $\left(I-P_{h}\right)$ to verify (4.38)

$$
\begin{aligned}
\left\|\left(I-P_{h}\right) u_{\beta}\right\|_{L^{\infty}\left(0, T, H^{-1}(\Omega)\right)} & =\left\|\left(I-P_{h}\right)^{2} u_{\beta}\right\|_{L^{\infty}\left(H^{-1}\right)} \\
& \leqslant C h\left\|\left(I-P_{h}\right) u_{\beta}\right\|_{L^{\infty}\left(L^{2}\right)} \leqslant C h^{1+1 /(1+\nu)}=C h^{\gamma} .
\end{aligned}
$$

LEMMA 4.7. For $0 \leqslant \beta \leqslant 1$,

$$
\left\|u-U_{h}\right\|_{L^{2+\nu}\left(0, T, L^{2+\nu}(\Omega)\right)}^{2+\nu} \leqslant\left\|\left(T-T_{h}\right) \partial u_{\beta} / \partial t\right\|_{L^{\gamma}\left(0, T, L^{\gamma}(\Omega)\right)}^{\gamma}+C \beta^{2+\nu} .
$$

If we assume the quasiuniformity hypothesis (4.2), then

$$
\begin{aligned}
\left\|u-P_{h} U_{h}\right\|_{L^{\infty}\left(H^{-1}\right)}^{2}+ & \left\|u-U_{h}\right\|_{L^{2+\nu}\left(L^{2+\nu}\right)}^{2+\nu} \\
& \leqslant C\left\{\left\|\left(T-T_{h}\right)\left(\partial u_{\beta} / \partial t\right)\right\|_{L^{\gamma}\left(L^{\gamma}\right)}^{\gamma}+h^{2 \gamma}+\beta^{2+\gamma}\right\}
\end{aligned}
$$

and

$$
\begin{aligned}
\left\|u-P_{h} U_{h}\right\|_{L^{\infty}\left(L^{2}\right)} & \leqslant C h^{-1}\left\|P_{h} u-P_{h} U_{h}\right\|_{L^{\infty}\left(H^{-1}\right)}+C h^{1 /(1+\nu)} \\
& \leqslant C h^{-1}\left\|u-P_{h} U_{h}\right\|_{L^{\infty}\left(H^{-1}\right)}+C h^{1 /(1+\nu)}
\end{aligned}
$$

Proof. Combine (2.15), (4.3), and (4.32) to obtain

$$
\begin{aligned}
\| u- & u_{\beta}\left\|_{L^{\infty}\left(H^{-1}\right)}^{2}+\right\| P_{h} u_{\beta}-P_{h} U_{h} \|_{L^{\infty}\left(H^{-1}\right)}^{2} \\
& +\int_{0}^{T}\left(K(u)-K\left(u_{\beta}\right), u-u_{\beta}\right)+\left(K_{\beta}\left(u_{\beta}\right)-K_{\beta}\left(U_{h}\right), u_{\beta}-U_{h}\right) d t \\
& \leqslant C\left\|\left(T-T_{h}\right) \frac{\partial u_{\beta}}{\partial t}\right\|_{L^{\gamma}\left(L^{\gamma}\right)}^{\gamma}+C \beta^{2+\nu} .
\end{aligned}
$$


By (2.22) and (2.32),

$$
\begin{aligned}
\text { (4.46) } \| u- & U_{h}\left\|_{L^{2+\nu}\left(L^{2+\nu}\right)}^{2+\nu} \leqslant C\right\| u-u_{\beta}\left\|_{L^{2+\nu}\left(L^{2+\nu}\right)}^{2+\nu}+C\right\| u_{\beta}-U_{h} \|_{L^{2+\nu}\left(L^{2+\nu}\right)}^{2+\nu} \\
& \leqslant C \int_{0}^{T}\left(K(u)-K\left(u_{\beta}\right), u-u_{\beta}\right)+\left(K_{\beta}\left(u_{\beta}\right)-K_{\beta}\left(U_{h}\right), u_{\beta}-U_{h}\right) d t .
\end{aligned}
$$

Bounds (4.45) and (4.46) imply (4.42).

If we assume (4.2), (4.25) yields

$$
\left\|P_{h} u_{\beta}-P_{h} U_{h}\right\|_{L^{\infty}\left(H^{-1}\right)} \leqslant C\left\|P_{h} u_{\beta}-P_{h} U_{h}\right\|_{L^{\infty}\left(H_{h}^{-1}\right)} \text {. }
$$

Combine (4.38), (4.45), and (4.47) to obtain (4.43). Finally, use (4.43), the inverse hypothesis (4.3), and (4.37) to obtain (4.44)

$$
\begin{aligned}
\left\|u-P_{h} U_{h}\right\|_{L^{\infty}\left(L^{2}\right)} & \leqslant\left\|\left(I-P_{h}\right) u\right\|_{L^{\infty}\left(L^{2}\right)}+\left\|P_{h} u-P_{h} U_{h}\right\|_{L^{\infty}\left(L^{2}\right)} \\
& \leqslant C h^{1 /(1+\nu)}+C h^{-1}\left\|P_{h} u-P_{h} U_{h}\right\|_{L^{\infty}\left(H^{-1}\right)} .
\end{aligned}
$$

To establish Theorems 4.1 through 4.3 we will need some additional results for the operator $T-T_{h}$.

Lemma 4.8. Let $T$ be defined by (2.10)-(2.13), and let $T_{h}$ be as in (4.20). Assume that the triangulation $\left\{\Delta_{h}\right\}$ is quasi-uniform, as in (4.2). Then, in one space dimension,

$$
\left\|\left(T-T_{h}\right) f\right\|_{L^{p}(\Omega)} \leqslant C h^{2}\|f\|_{L^{p}(\Omega)}
$$

for all $f \in L^{p}(\Omega), 1 \leqslant p \leqslant \infty$. When $\operatorname{dim}(\Omega)=2$ or 3 , we have

$$
\left\|\left(T-T_{h}\right) f\right\|_{L^{p}(\Omega)} \leqslant C[\ln (1 / h)]^{|1-2 / p|}\|f\|_{L^{p}(\Omega)}
$$

for all $f \in L^{p}(\Omega), 1<p<\infty$.

Proof. The estimate

$$
\left\|\left(I-E_{h}\right) \phi\right\|_{L^{p}(\Omega)} \leqslant C[\ln (1 / h)]^{\alpha|1-2 / p|} h^{2}\|\phi\|_{W^{2, p}(\Omega)}
$$

is valid for $1 \leqslant p \leqslant \infty$ with $\alpha=0$ when $\operatorname{dim}(\Omega)=1$ and $\alpha=1$ for $\operatorname{dim}(\Omega)=2$ or 3. The one-dimensional case was proved by Douglas, Dupont, and Wahlbin [9]. For two space dimensions, (4.50) was verified by Nitsche [13] and Scott [20]. The three-dimensional case is treated in Ciarlet [7] and Nitsche [14].

To complete the argument, we invoke the elliptic regularity result [7]

$$
\|T \phi\|_{W^{2, p}(\Omega)} \leqslant C\|\phi\|_{L^{p}(\Omega)}
$$

which is valid for $1 \leqslant p \leqslant \infty$ when $\operatorname{dim}(\Omega)=1$ and for $1<p<\infty$ when $\operatorname{dim}(\Omega)>$ 1.

Proof of Theorem 4.1. When $\nu=1$ and $\operatorname{dim}(\Omega) \geqslant 1$, estimate (3.15) implies

$$
\left\|\partial u_{\beta} / \partial t\right\|_{L^{2}\left(0, T, L^{2}(\Omega)\right)} \leqslant C\left\|\partial u_{\beta} / \partial t\right\|_{L^{x}\left(0, T, L^{3}(\Omega)\right)} \leqslant C_{11} .
$$

By (4.31), we have

$$
\begin{aligned}
\|(T- & \left.T_{h}\right) \partial u_{\beta} / \partial t\left\|_{L^{\gamma}\left(L^{\gamma}\right)}^{\gamma} \leqslant C\right\|\left(T-T_{h}\right) \partial u_{\beta} / \partial t \|_{L^{\gamma}\left(L^{\gamma}\right)} \\
& \leqslant C\left(h^{2}\left\|\partial u_{\beta} / \partial t\right\|_{L^{2}\left(L^{2}\right)}\right)^{\gamma}=C h^{2 \gamma}\left\|\partial u_{\beta} / \partial t\right\|_{L^{2}\left(L^{2}\right)}^{\gamma} \leqslant C h^{2 \gamma} .
\end{aligned}
$$


When $1 \leqslant \nu<2$ and $\operatorname{dim}(\Omega)=1$, estimate (3.43) or bound (3.55) implies

$$
\left\|\partial u_{\beta} / \partial t\right\|_{L^{2}\left(0, T, L^{2}(\Omega)\right)} \leqslant C_{12}
$$

so that (4.52) holds.

When $\operatorname{dim}(\Omega)=1$ and $\nu \geqslant 2$, we can use (4.49) with $\alpha=0$ and $p=\gamma$ together with (3.56) to obtain

$$
\begin{aligned}
\left\|\left(T-T_{h}\right) \partial u_{\beta} / \partial t\right\|_{L^{\gamma}\left(L^{\gamma}\right)}^{\gamma} & \leqslant\left(C h^{2}\left\|\partial u_{\beta} / \partial t\right\|_{L^{\gamma}\left(L^{\gamma}\right)}\right)^{\gamma} \\
& \leqslant C h^{2 \gamma}\left\|\partial u_{\beta} / \partial t\right\|_{L^{\gamma}\left(L^{\gamma}\right)}^{\gamma} \leqslant C_{13} .
\end{aligned}
$$

Use (4.52) or (4.54) with $0 \leqslant \beta \leqslant C h^{2 /(1+\nu)}$ to prove (4.9). Use (4.43) and (4.48) to verify (4.8) under the quasiuniformity assumption (4.2). Combine (4.44) and (4.52) or (4.54) to establish (4.10).

Proof of Theorem 4.2. By estimates (4.48a) and (4.48b),

$$
\begin{aligned}
\left\|\left(T-T_{h}\right) u_{\beta t}\right\|_{L^{\gamma}\left(0, T, L^{\gamma}(\Omega)\right)}^{\gamma} & \leqslant C\left([\ln (1 / h)]^{\alpha|1-2 / \gamma|} h^{2}\left\|u_{\beta t}\right\|_{L^{\gamma}\left(0, T, L^{\gamma}(\Omega)\right)}\right)^{\gamma} \\
& =C[\ln (1 / h)]^{\alpha \nu /(1+\nu)} h^{2 \gamma}\left\|u_{\beta t}\right\|_{L^{\gamma}\left(0, T, L^{\gamma}(\Omega)\right)}^{\gamma},
\end{aligned}
$$

where $\alpha=0$ if $\operatorname{dim}(\Omega)=1$ and $\alpha=1$ if $\operatorname{dim}(\Omega)=2$ or 3. By Hölder's inequality and the Riesz-Thorin interpolation theorem [21]

$$
\begin{aligned}
\left\|u_{\beta t}\right\|_{L^{\gamma}\left(0, T, L^{\gamma}(\Omega)\right)}^{\gamma} & \leqslant C \int_{0}^{T}\left\|u_{\beta t}\right\|_{L^{\prime}(\Omega)}^{\nu /(\nu+1)}\left\|u_{\beta t}\right\|_{L^{2}(\Omega)}^{2 /(\nu+1)} d t \\
& \leqslant C\left\|u_{\beta t}\right\|_{L^{\prime}\left(0, T, L^{\prime}(\Omega)\right)}^{\nu /(\nu+1)}\left\|u_{\beta t}\right\|_{L^{2}\left(0, T, L^{2}(\Omega)\right)}^{2 /(\nu+1)},
\end{aligned}
$$

where the first factor on the right is known to be bounded independent of $\beta$ by Theorem 3.2. To bound the second factor, use (2.26) and (3.1) to see that

$$
\left\|u_{\beta t}\right\|_{L^{2}\left(L^{2}\right)} \leqslant c \beta^{-\nu / 2} \text {. }
$$

Combine (4.55), (4.56), and (4.57) to obtain

$$
\left\|\left(T-T_{h}\right) u_{\beta t}\right\|_{L^{\gamma}\left(L^{\gamma}\right)}^{\gamma} \leqslant C[\ln (1 / h)]^{\alpha \nu /(1+\nu)} h^{2 \gamma} \beta^{-\nu /(1+\nu)} .
$$

Use (4.42) and (4.58) to see that

$$
\left\|u-U_{h}\right\|_{L^{2+\nu}\left(L^{2+\nu}\right)}^{2+\nu} \leqslant C\left([\ln (1 / h)]^{\alpha} h^{2 \gamma} \beta^{-\nu}+\beta^{2+\nu}\right),
$$

and choose $\beta=h^{\sigma}$ as in (4.11) to obtain (4.13). Use (4.43) and (4.58) to verify (4.12), and combine (4.44) and (4.58) to prove (4.14).

Proof of Theorem 4.3. If we assume (4.2) and (4.15), bound (4.48) with $p=\gamma$ yields

$$
\left\|\left(T-T_{h}\right) u_{\beta t}\right\|_{L^{\gamma}\left(L^{\gamma}\right)}^{\gamma} \leqslant C[\ln (1 / h)]^{\alpha \nu /(1+\nu)} h^{2 \gamma},
$$

where $\alpha=0$ if $\operatorname{dim}(\Omega)=1$ and $\alpha=1$ when $\operatorname{dim}(\Omega)=2$ or 3. Combine (4.60) with (4.42), and suppose $0 \leqslant \beta \leqslant C h^{2 /(1+\nu)}$ to prove (4.17). Use (4.43) and (4.60) to verify (4.16), and combine (4.44) with (4.60) to prove (4.18). Under the regularity hypothesis (4.19), the proof of Theorem 4.1 indicates that (4.16)-(4.18) are valid for $\nu \geqslant 2$ with $\alpha=0$ for $\operatorname{dim}(\Omega)=1,2$, or 3 . 
5. Backward-Difference Schemes. Let the triangulation $\left\{\Delta_{h}\right\}$ and the finite element spaces $\left\{M_{h}\right\}$ be those defined in Section 4 for $0<h \leqslant 1$. Let $\Delta t=T / N$, where $N$ is a positive integer, and define $t_{n}=n \cdot \Delta t$ for $n=0,1, \ldots, N$. For a function $F$ on $[0, T]$, let $F^{n}$ denote $F\left(t_{n}\right)$, and define $\left(\partial^{+} F\right)^{n}=\left(F^{n+1}-F^{n}\right) / \Delta t$.

Our backward-difference scheme consists of finding $V_{h}^{n} \in M_{h}, n=0,1, \ldots, N$, the solution of the nonlinear algebraic equations

$$
\left(\left(\partial^{+} H_{\beta}\left(V_{h}\right)\right)^{n}, \chi\right)+\left(\nabla V_{h}^{n+1}, \nabla \chi\right)=0
$$

for $\chi \in M_{h}$ and $n=0,1, \ldots, N-1$, with the initial function defined by

$$
P_{h} H_{\beta}\left(V_{h}^{0}\right)=P_{h} u_{0} .
$$

The parameter $\beta$ will be given below. The existence and uniqueness of $U_{h}^{n}=H_{\beta}\left(V_{h}^{n}\right)$ may be proved using elementary monotone operator theory [6]. We may rewrite $(5.1)-(5.2)$ as

$$
\left(\left(\partial^{+} U_{h}\right)^{n}, \chi\right)+\left(\nabla K_{\beta}\left(U_{h}^{n+1}\right), \nabla \chi\right)=0
$$

for $\chi \in M_{h}$ and $n=0,1, \ldots, N-1$, and

$$
P_{h} U_{h}=P_{h} u_{0} .
$$

THEOREM 5.1. Suppose $\operatorname{dim}(\Omega)=1$ and $\nu<2$ or that $\operatorname{dim}(\Omega)>1$ and $\nu=1$. Let $0 \leqslant \beta \leqslant C h^{2 /(\nu+1)}$, and let $U_{h}^{n}=H_{\beta}\left(U_{h}^{n}\right)$, where $U_{h}^{n}$ solves (5.1)-(5.2) for $n=$ $0,1, \ldots, N$. Then for $\operatorname{dim}(\Omega)=1,2$, or 3 ,

$$
\left(\sum\left\|\left(u-U_{h}\right)^{n}\right\|_{L^{2+\nu}(\Omega)}^{2+\nu} \cdot \Delta t\right)^{1 /(2+\nu)} \leqslant C\left(h^{2 /(1+\nu)}+(\Delta t)^{2 /(2+\nu)}\right) .
$$

If we assume (4.2), then

$$
\operatorname{Max}_{n}\left\|u^{n}-P_{h} U_{h}^{n}\right\|_{H^{-1}(\Omega)} \leqslant C\left(h^{\gamma}+(\Delta t)\right),
$$

and, if we also require $\Delta t \leqslant C h^{\gamma}$,

$$
\operatorname{Max}_{n}\left\|u^{n}-P_{h} U_{h}^{n}\right\|_{L^{2}(\Omega)} \leqslant C\left(h^{\gamma-1}+h^{-1}(\Delta t)\right)=C h^{1 /(1+\nu)} .
$$

TheOREM 5.2. Suppose $\operatorname{dim}(\Omega)=1$ and $\nu_{1} \geqslant 2$. Then

$$
\left(\sum_{n=0}^{N}\left\|\left(u-U_{h}^{n}\right)\right\|_{L^{2+\nu}(\Omega)}^{2+\nu} \cdot \Delta t\right)^{1 /(2+\nu)} \leqslant C\left(h^{2 /(1+\nu)}+(\Delta t)^{1 /(1+\nu)}\right) .
$$

Under the quasiuniformity assumption (4.2)

$$
\operatorname{Max}_{n}\left\|u^{n}-P_{h} U_{h}^{n}\right\|_{H^{-1}(\Omega)} \leqslant C\left(h^{\gamma}+(\Delta t)^{\gamma / 2}\right),
$$

and, if we assume $\Delta t \leqslant C h^{2}$,

$$
\operatorname{Max}_{n}\left\|u^{n}-P_{h} U_{h}^{n}\right\|_{L^{2}(\Omega)} \leqslant C\left(h^{\gamma-1}+h^{-1}(\Delta t)^{\gamma / 2}\right)=C h^{1 /(1+\nu)} .
$$

When $\operatorname{dim}(\Omega)>1$ and $\nu>1$, the known convergence rates are probably not sharp. 
TheOREM 5.3. Suppose (4.2) holds, let $\beta$ be given by (4.11), let $\nu>1$, and let $\operatorname{dim}(\Omega)=2$ or 3 . Then

$$
\begin{aligned}
& \left(\sum_{n=0}^{N}\left\|\left(u-U_{h}\right)^{n}\right\|_{L^{2+\nu}(\Omega)}^{2+\nu} \cdot \Delta t\right)^{1 /(2+\nu)} \\
& \quad \leqslant C\left([\ln (1 / h)]^{\alpha \nu / 2(1+\nu)} h^{(2+\nu) / 2 \cdot \sigma}+(\Delta t)^{\gamma / 2}\right),
\end{aligned}
$$

$$
\operatorname{Max}_{n}\left\|\left(u-P_{h} U_{h}\right)^{n}\right\|_{H^{-1}(\Omega)} \leqslant C\left([\ln (1 / h)]^{\alpha \nu / 2(1+\nu)} h^{(2+\nu) / 2 \cdot \sigma}+(\Delta t)^{\gamma / 2}\right),
$$

and, assuming $\Delta t \leqslant C h^{(1+\nu) \sigma}$,

$$
\operatorname{Max}_{n}\left\|\left(u-P_{h} U_{h}\right)^{n}\right\|_{L^{2}(\Omega)} \leqslant C\left([\ln (1 / h)]^{\alpha \nu /(2(1+\nu))} h^{((2+\nu) / 2) \cdot \sigma-1}\right),
$$

where $\alpha=0$ if $\operatorname{dim}(\Omega)=1$ and $\alpha=1$ if $\operatorname{dim}(\Omega)=2$ or 3 .

THEOREM 5.4. Suppose (4.2)and (4.15) are valid. Suppose $0 \leqslant \beta \leqslant C h^{2 /(1+v)}$, and let $\alpha=0$ if $\operatorname{dim}(\Omega)=1$ and $\alpha=1$ if $\operatorname{dim}(\Omega)=2$ or 3 . Then

$$
\begin{gathered}
\left(\sum_{n=0}^{N}\left\|\left(u-U_{h}\right)^{n}\right\|_{L^{2+\nu}(\Omega)}^{2+\nu} \Delta t\right)^{1 /(2+\nu)} \\
\leqslant C\left([\ln (1 / h)]^{\alpha /(1+\nu)(2+\nu)} h^{2 /(1+\nu)}+(\Delta t)^{1 /(1+\nu)}\right), \\
\operatorname{Max}_{n}\left\|\left(u-P_{h} U_{h}\right)^{n}\right\|_{H^{-1}(\Omega)} \leqslant C\left([\ln (1 / h)]^{\alpha \nu /(2+\nu)} h^{\gamma}+(\Delta t)^{\gamma / 2}\right),
\end{gathered}
$$

and, assuming $\Delta t \leqslant C h^{2}$,

$$
\operatorname{Max}_{n}\left\|\left(u-P_{h} U_{h}\right)^{n}\right\|_{L^{2}(\Omega)} \leqslant C\left([\ln (1 / h)]^{\alpha /(2 \nu+2)} h^{1 /(\nu+1)}+(\Delta t)^{1 /(2 \nu+2)}\right) .
$$

If (4.19) is valid, then (5.13)-(5.15) hold with $\alpha=0$ for $\operatorname{dim}(\Omega)=1,2$, or 3 . Moreover, when (3.47) holds, then bounds (5.5)-(5.6) are still valid.

To prove these results, we shall need to modify Theorem 2.1 .

LEMMA 5.5. Let $u_{\beta}$ be the solution of (2.3)-(2.5). Then

$$
\begin{gathered}
\operatorname{Max}_{n}\left\|u_{\beta}^{n}-u^{n}\right\|_{H^{-1}(\Omega)}^{2}+\sum_{n=0}^{N}\left(K_{\beta}\left(u_{\beta}\right)^{n}-K_{\beta}(u)^{n},\left(u_{\beta}-u\right)^{n}\right) \cdot \Delta t \\
\leqslant C\left(\beta^{2+\nu}+(\Delta t)^{\gamma}\right) .
\end{gathered}
$$

When (3.47) is valid, a stronger result is true

$$
\begin{gathered}
\operatorname{Max}_{n}\left\|u_{\beta}^{n}-u^{n}\right\|_{H^{-1}(\Omega)}^{2}+\sum_{n=0}^{N}\left(K_{\beta}\left(u_{\beta}\right)^{n}-K_{\beta}(u)^{n},\left(u_{\beta}-u\right)^{n}\right) \cdot \Delta t \\
\leqslant C\left(\beta^{2+\nu}+(\Delta t)^{2}\right) .
\end{gathered}
$$

Proof. Equation (2.16) can be rewritten as

$$
\begin{aligned}
\left(\partial^{+} u\right)^{n} & =\Delta K(u)^{n+1}-\frac{1}{\Delta t} \int_{t_{n}}^{t_{n+1}}\left(u_{t}\left(t_{n+1}\right)-u_{t}(s)\right) d s \\
& =\Delta K(u)^{n+1}-\frac{1}{\Delta t} \int_{t_{n}}^{t_{n+1}} \int_{s}^{t_{n+1}} u_{t t}(\tau) d \tau d s
\end{aligned}
$$


and (2.17) can be expressed as

$$
\left(\partial^{+} u_{\beta}\right)^{n}=\Delta K_{\beta}\left(u_{\beta}\right)^{n+1}-\frac{1}{\Delta t} \int_{t_{n}}^{t_{n+1}} \int_{s}^{t_{n+1}} u_{\beta t t}(\tau) d \tau d s
$$

Subtract (5.18) from (5.19), integrate the difference against $T\left(u_{\beta}-u\right)^{n+1}$, and use the Cauchy-Schwarz inequality to obtain

$$
\begin{aligned}
\frac{1}{2 \Delta t}\left\{\left\|\left(u_{\beta}-u\right)^{n+1}\right\|_{H^{-1}(\Omega)}^{2}-\left\|\left(u_{\beta}-u\right)^{n}\right\|_{H^{-1}(\Omega)}^{2}\right\} & +\left(K_{\beta}\left(u_{\beta}\right)^{n+1}-K_{\beta}(u)^{n+1},\left(u_{\beta}-u\right)^{n+1}\right) \\
\leqslant & \left(K(u)^{n+1}-K_{\beta}(u)^{n+1},\left(u_{\beta}-u\right)^{n+1}\right) \\
& -\left(\frac{1}{\Delta t} \int_{t_{n}}^{t_{n+1}} \int_{s}^{t_{n+1}}\left(u_{\beta}-u\right)_{t t}(\tau) d \tau d s, T\left(u_{\beta}-u\right)^{n+1}\right)
\end{aligned}
$$

where we have used the fact that

$$
\int_{\Omega}\left(u_{\beta}-u\right)^{n} d x=0, \quad n=0,1, \ldots, N
$$

By Hölder's inequality, (2.24), and (2.33), the first term on the right side of (5.20) may be bounded by

$$
\begin{aligned}
& \left\|K(u)^{n+1}-K_{\beta}(u)^{n+1}\right\|_{L^{\gamma}(\Omega)}\left\|\left(u_{\beta}-u\right)^{n+1}\right\|_{L^{2+\nu}(\Omega)} \\
& \quad \leqslant C\left\|K(u)^{n+1}-K_{\beta}(u)^{n+1}\right\|_{L^{\gamma}(\Omega)}^{\gamma}+\frac{\eta}{4}\left\|\left(u_{\beta}-u\right)^{n+1}\right\|_{L^{2+\nu}(\Omega)}^{2+\nu} \\
& \quad \leqslant C \beta^{2+\nu}+\frac{1}{4}\left(K_{\beta}\left(u_{\beta}\right)^{n+1}-K_{\beta}(u)^{n+1},\left(u_{\beta}-u\right)^{n+1}\right) .
\end{aligned}
$$

As for the second term on the right side of (5.20), use Hölder's inequality (2.24), and (2.33) to see that

$$
\begin{aligned}
&-\left(\frac{1}{\Delta t} \int_{t_{n}}^{t_{n+1}} \int_{s}^{t_{n+1}}\left(u_{\beta}-u\right)_{t t}(\tau) d \tau d s, T\left(u_{\beta}-u\right)^{n+1}\right) \\
&=-\left(\frac{1}{\Delta t} \int_{t_{n}}^{t_{n+1}} \int_{s}^{t_{n+1}} T\left(u_{\beta}-u\right)_{t t}(\tau) d \tau d s,\left(u_{\beta}-u\right)^{n+1}\right) \\
& \leqslant \frac{1}{\Delta t} \int_{t_{n}}^{t_{n+1}} \int_{s}^{t_{n+1}}\left\|T\left(u_{\beta}-u\right)(\tau)\right\|_{L^{\gamma}(\Omega)} d \tau d s \cdot\left\|\left(u_{\beta}-u\right)^{n+1}\right\|_{L^{2+\nu}(\Omega)} \\
& \leqslant C\left(\frac{1}{\Delta t} \int_{t_{n}}^{t_{n+1}} \int_{s}^{t_{n+1}}\left\|T\left(u_{\beta}-u\right)(\tau)\right\|_{L^{\gamma}(\Omega)} d \tau d s\right)^{\gamma} \\
&+\frac{\eta}{4}\left\|\left(u_{\beta}-u\right)^{n+1}\right\|_{L^{2+\nu}(\Omega)}^{2+\nu} \\
& \leqslant C\left(\frac{1}{\Delta t} \int_{t_{n}}^{t_{n+1}} \int_{s}^{t_{n+1}}\left\|T\left(u_{\beta}(\tau)\right)\right\|_{L^{\gamma}(\Omega)} d \tau d s\right)^{\gamma} \\
&+C\left(\frac{1}{\Delta t} \int_{t_{n}}^{t_{n+1}} \int_{s}^{t_{n+1}}\|T(u(\tau))\|_{L^{\gamma}(\Omega)} d \tau d s\right)^{\gamma} \\
&+\frac{1}{4}\left(K_{\beta}\left(u_{\beta}\right)^{n+1}-K_{\beta}(u)^{n+1},\left(u_{\beta}-u\right)^{n+1}\right)
\end{aligned}
$$

where the last term may be hidden on the left side of (5.20). 
By the Jensen and Hölder inequalities,

$$
\begin{aligned}
\left(\frac{1}{\Delta t} \int_{t_{n}}^{t_{n+1}} \int_{s}^{t_{n+1}}\left\|T u_{\beta t t}(\tau)\right\|_{L^{\gamma}(\Omega)} d \tau d s\right)^{\gamma} & \\
& \leqslant \frac{C}{\Delta t} \int_{t_{n}}^{t_{n+1}}\left(\int_{s}^{t_{n+1}}\left\|T u_{\beta t t}(\tau)\right\|_{L^{\gamma}(\Omega)} d \tau\right)^{\gamma} d s \\
& \leqslant \frac{C}{\Delta t} \int_{t_{n}}^{t_{n+1}}\left(\left(t_{n+1}-s\right)^{1 /(2+\nu)}\left\|T u_{\beta t t}\right\|_{L^{\gamma}\left(s, t_{n+1}, L^{\gamma}(\Omega)\right)}\right)^{\gamma} d s \\
& \leqslant C(\Delta t)^{1 /(1+\nu)}\left\|T u_{\beta t t}\right\|_{L^{\gamma}\left(t_{n}, t_{n+1}, L^{\gamma}(\Omega)\right)}^{\gamma}
\end{aligned}
$$

Setting $\beta=0$, we also have

$$
\left(\frac{1}{\Delta t} \int_{t_{n}}^{t_{n+1}} \int_{s}^{t_{n+1}}\left\|T u_{t t}(\tau)\right\|_{L^{\gamma}(\Omega)} d \tau d s\right)^{\gamma} \leqslant C(\Delta t)^{1 /(1+\nu)}\left\|T u_{t t}\right\|_{L^{\gamma}\left(t_{n}, t_{n+1}, L^{\gamma}(\Omega)\right)}^{\gamma} .
$$

By (5.20)-(5.24),

$$
\begin{aligned}
& \frac{1}{2 \Delta t}\left\{\left\|\left(u_{\beta}-u\right)^{n+1}\right\|_{H^{-1}(\Omega)}^{2}-\left\|\left(u_{\beta}-u\right)^{n}\right\|_{H^{-1}(\Omega)}^{2}\right\} \\
& \quad+\frac{1}{2}\left(\left(K_{\beta}\left(u_{\beta}\right)^{n+1}-K_{\beta}(u)\right)^{n+1},\left(u_{\beta}-u\right)^{n+1}\right) \\
& \leqslant C \beta^{2+\nu}+C(\Delta t)^{1 /(1+\nu)}\left\{\left\|T u_{\beta t t}\right\|_{L^{\gamma}\left(t_{n}, t_{n+1}, L^{\gamma}(\Omega)\right)}^{\gamma}+\left\|T u_{t t}\right\|_{L^{\gamma}\left(t_{n}, t_{n+1}, L^{\gamma}(\Omega)\right)}^{\gamma}\right\}
\end{aligned}
$$

Multiply by $\Delta t$, and sum on $n$ to obtain

$$
\begin{array}{r}
\operatorname{Max}_{n}\left\|\left(u_{\beta}-u\right)^{n}\right\|_{H^{-1}(\Omega)}^{2}+\sum_{n}\left(K_{\beta}\left(u_{\beta}\right)^{n+1}-K_{\beta}(u)^{n+1},\left(u_{\beta}-u\right)^{n+1}\right) \cdot \Delta t \\
\leqslant C \beta^{2+\nu}+C(\Delta t)^{\gamma}\left\{\left\|T u_{\beta t t}\right\|_{L^{\gamma}\left(0, T, L^{\gamma}(\Omega)\right)}^{\gamma}+\left\|T u_{t t}\right\|_{L^{\gamma}\left(0, T, L^{\gamma}(\Omega)\right)}^{\gamma}\right\} .
\end{array}
$$

Recall bound (3.3)

$$
\left\|K_{\beta}\left(u_{\beta}\right)_{t}\right\|_{L^{2}\left(0, T, L^{2}(\Omega)\right)} \leqslant C, \quad 0 \leqslant \beta \leqslant 1
$$

and note that

$$
\begin{aligned}
\left\|T u_{\beta t t}\right\|_{L^{\gamma}\left(L^{\gamma}\right)} & =\left\|K_{\beta}\left(u_{\beta}\right)_{t}-\frac{1}{|\Omega|} \int_{\Omega} K_{\beta}\left(u_{\beta}\right)_{t} d x\right\|_{L^{\gamma}\left(L^{\gamma}\right)} \\
& \leqslant\left\|K_{\beta}\left(u_{\beta}\right)_{t}\right\|_{L^{\gamma}\left(L^{\gamma}\right)} \leqslant C\left\|K_{\beta}\left(u_{\beta}\right)_{t}\right\|_{L^{2}\left(L^{2}\right)} \leqslant C
\end{aligned}
$$

for $0 \leqslant \beta \leqslant 1$. This completes the proof of (5.16) in the former case.

Next, suppose (3.47) holds

$$
\left\|\nabla K_{\beta}\left(u_{\beta}\right)_{t}\right\|_{L^{2}\left(0, T, L^{2}(\Omega)\right)} \leqslant C, \quad 0 \leqslant \beta \leqslant 1 .
$$

Use the representation

$$
-T\left(u_{\beta}-u\right)_{t t}=K_{\beta}\left(u_{\beta}\right)_{t}-K(u)_{t}-\frac{1}{|\Omega|} \int_{\Omega} K_{\beta}\left(u_{\beta}\right)_{t}-K_{\beta}\left(u_{\beta}\right)_{t} d x
$$


and the Cauchy-Schwarz inequality to bound the second term on the right side of (5.20) by

$$
\begin{aligned}
&\left(\frac{1}{\Delta t} \int_{t_{n}}^{t_{n+1}} \int_{s}^{t_{n+1}} T\left(u_{\beta}-u\right)_{t t}(\tau) d \tau d s,\left(u_{\beta}-u\right)^{n+1}\right) \\
& \leqslant \frac{1}{\Delta t} \int_{t_{n}}^{t_{n+1}} \int_{s}^{t_{n+1}}\left\|T\left(u_{\beta}-u\right)_{t t}(\tau)\right\|_{H^{1}(\Omega)} d \tau d s \cdot\left\|\left(u_{\beta}-u\right)^{n+1}\right\|_{H^{-1}(\Omega)} \\
& \leqslant \frac{1}{\Delta t} \int_{t_{n}}^{t_{n+1}} \int_{s}^{t_{n+1}}\left(\left\|\nabla K_{\beta}\left(u_{\beta}\right)_{t}(\tau)\right\|+\left\|\nabla K(u)_{t}(\tau)\right\|\right) d t d s \\
& \cdot\left\|\left(u_{\beta}-u\right)^{n+1}\right\|_{H^{-1}(\Omega)} \\
& \leqslant C \cdot \sqrt{\Delta t} \cdot\left(\left\|\nabla K_{\beta}\left(u_{\beta}\right)_{t}\right\|_{L^{2}\left(t_{n}, t_{n+1}, L^{2}(\Omega)\right)}+\left\|\nabla K(u)_{t}\right\|_{L^{2}\left(t_{n}, t_{n+1}, L^{2}(\Omega)\right)}\right) \\
& \quad \cdot\left\|\left(u_{\beta}-u\right)^{n+1}\right\|_{H^{-1}(\Omega)} \\
& \leqslant C \Delta t \cdot\left(\left\|\nabla K_{\beta}\left(u_{\beta}\right)_{t}\right\|_{L^{2}\left(t_{n}, t_{n+1}, L^{2}(\Omega)\right)}^{2}+\left\|\nabla K(u)_{t}\right\|_{L^{2}\left(t_{n}, t_{n+1}, L^{2}(\Omega)\right)}^{2}\right) \\
&+C\left\|\left(u_{\beta}-u\right)^{n+1}\right\|_{H^{-1}(\Omega)}^{2}
\end{aligned}
$$

Bounds (5.20), (5.21), and (5.28) yield

$$
\begin{gathered}
\frac{1}{2 \Delta t}\left\{\left\|\left(u_{\beta}-u\right)^{n+1}\right\|_{H^{-1}(\Omega)}^{2}-\left\|\left(u_{\beta}-u\right)^{n}\right\|_{H^{-1}(\Omega)}^{2}\right\} \\
+\frac{1}{2}\left(K_{\beta}\left(u_{\beta}\right)^{n+1}-K_{\beta}(u)^{n+1},\left(u_{\beta}-u\right)^{n+1}\right) .
\end{gathered}
$$

Multiply (5.29) by $2 \Delta t$, sum on $n$, apply the discrete Gronwall lemma, and appeal to (3.47) to verify $(5.16)$

$$
\begin{gathered}
\operatorname{Max}_{n}\left\|\left(u_{\beta}-u\right)^{n}\right\|_{H^{-1}(\Omega)}^{2}+\sum_{n}\left(K_{\beta}\left(u_{\beta}\right)^{n+1}-K_{\beta}(u)^{n+1},\left(u_{\beta}-u\right)^{n+1}\right) \cdot \Delta t \\
\quad \leqslant C \beta^{2+\nu}+C(\Delta t)^{2}\left(\left\|\nabla K_{\beta}\left(u_{\beta}\right)\right\|_{t}\left\|_{L^{2}\left(0, T, L^{2}(\Omega)\right)}^{2}+\right\| \nabla K(u)_{t} \|_{L^{2}\left(0, T, L^{2}(\Omega)\right)}^{2}\right) \\
\quad \leqslant C \beta^{2+\nu}+C(\Delta t)^{2} .
\end{gathered}
$$

LEMMA 5.6. Let $U_{h}^{n}=H_{\beta}\left(V_{h}^{n}\right)$, where $V_{h}^{n}, n=0,1, \ldots, N$, solves (5.1)-(5.2). Then, with $m=\gamma=(2+\nu) /(1+\nu)$,

$$
\begin{gathered}
\operatorname{Max}_{n}\left\|\left(u_{\beta}-P_{h} U_{h}\right)^{n}\right\|_{H_{h}^{-1}}^{2}+\sum_{n}\left(K_{\beta}\left(u_{\beta}\right)^{n}-K_{\beta}\left(U_{h}\right)^{n},\left(u_{\beta}-U_{h}\right)^{n}\right) \cdot \Delta t \\
\leqslant C\left\|\left(T-T_{h}\right) \partial u_{\beta} / \partial t\right\|_{L^{\gamma}\left(L^{\gamma}\right)}^{\gamma}+C(\Delta t)^{m} .
\end{gathered}
$$

Moreover,

$$
\begin{aligned}
& \sum_{n}\left\|\left(u-U_{h}\right)^{n}\right\|_{L^{2+\nu}(\Omega)}^{2+\nu} \cdot \Delta t \\
& \quad \leqslant C\left\|\left(T-T_{h}\right) \partial u_{\beta} / \partial t\right\|_{L^{\gamma}\left(L^{\gamma}\right)}^{\gamma}+C(\Delta t)^{m}+C \beta^{2+\nu} .
\end{aligned}
$$


If we assume the quasiuniformity hypothesis (4.2), then

(5.32) $\operatorname{Max}_{n}\left\|\left(u-P_{h} U_{h}\right)^{n}\right\|_{H^{-1}(\Omega)}^{2} \leqslant C h^{-1} \operatorname{Max}_{n}\left\|\left(u-P_{h} U_{h}\right)^{n}\right\|_{H^{-1}(\Omega)}+C h^{1 /(1+\nu)}$

and

(5.33) $\operatorname{Max}_{n}\left\|\left(u-P_{h} U_{h}\right)^{n}\right\|_{L^{2}(\Omega)} \leqslant C h^{-1} \operatorname{Max}_{n}\left\|\left(u-P_{h} U_{h}\right)^{n}\right\|_{H^{-1}(\Omega)} \leqslant C h^{1 /(1+\nu)}$.

If (3.47) is valid, then (5.32) and (5.33) hold with $m=2$.

Proof. Use (5.3)-(5.4) and (5.19) to obtain

$$
\begin{aligned}
\left(\left(\partial^{+}\left(u_{\beta}-U_{h}\right)\right)^{n}, \chi\right) & +\left(\nabla\left(K_{\beta}\left(u_{\beta}\right)^{n+1}-K_{\beta}\left(U_{h}\right)^{n+1}\right), \nabla \chi\right) \\
& =-\left(\frac{1}{\Delta t} \int_{t_{n}}^{t_{n+1}} \int_{s}^{t_{n+1}} u_{\beta t t}(\tau) d \tau d s, \chi\right)
\end{aligned}
$$

Choose $\chi=T_{h}\left(u_{\beta}-U_{h}\right)^{n+1}$, and use the fact that $\left(u_{\beta}-U_{h}\right)^{n+1}$ has mean value zero on $\Omega$ to see that

$$
\begin{aligned}
\frac{1}{2 \Delta t}\left\{\left\|\left(u_{\beta}-U_{h}\right)^{n+1}\right\|_{H_{h}^{-1}}^{2}-\left\|\left(u_{\beta}-U_{h}\right)^{n}\right\|_{H_{h}^{-1}}^{2}\right\} \\
+\left(K_{\beta}\left(u_{\beta}\right)^{n+1}-K_{\beta}(u)^{n+1},\left(u_{\beta}-u\right)^{n+1}\right) \\
=-\left(\frac{1}{\Delta t} \int_{t_{n}}^{t_{n+1}} \int_{s}^{t_{n+1}} T u_{\beta t t}(\tau) d \tau d s,\left(u_{\beta}-U_{h}\right)^{n+1}\right) \\
-\left(\left(T-T_{h}\right)\left(\partial^{+} u_{\beta}\right)^{n},\left(u_{\beta}-U_{h}\right)^{n+1}\right) .
\end{aligned}
$$

Use Hölder's inequality, (2.24), and (5.23) to bound the first term on the right side of (5.35) by

$$
\begin{aligned}
& \frac{1}{\Delta t} \int_{t_{n}}^{t_{n+1}} \int_{s}^{t_{n+1}}\left\|T u_{\beta t t}(\tau)\right\|_{L^{\gamma}(\Omega)} d \tau d s \cdot\left\|\left(u_{\beta}-U_{h}\right)^{n+1}\right\|_{L^{2+\nu}(\Omega)} \\
& \leqslant C\left(\frac{1}{\Delta t} \int_{t_{n}}^{t_{n+1}} \int_{s}^{t_{n+1}}\left\|T u_{\beta t t}(\tau)\right\|_{L^{\gamma}(\Omega)} d \tau d s\right)^{\gamma}+\frac{\eta}{4}\left\|\left(u_{\beta}-U_{h}\right)^{n+1}\right\|_{L^{2+\nu}(\Omega)}^{2+\nu} \\
& \leqslant C(\Delta t)^{1 /(1+\nu)}\left\|T u_{\beta t t}\right\|_{L^{\gamma}\left(t_{n}, t_{n+1}, L^{\gamma}(\Omega)\right)}^{\gamma} \\
& \quad+\frac{1}{4}\left(K_{\beta}\left(u_{\beta}\right)^{n+1}-K_{\beta}\left(U_{h}\right)^{n+1},\left(u_{\beta}-U_{h}\right)^{n+1}\right)
\end{aligned}
$$

where the last term may be hidden.

Next, note that

$$
\begin{aligned}
\left\|\left(T-T_{h}\right)\left(\partial^{+} u_{\beta}\right)^{n}\right\|_{L^{\gamma}(\Omega)} & =\frac{1}{\Delta t} \int_{t_{n}}^{t_{n+1}}\left\|\left(T-T_{h}\right) u_{\beta t}(\tau)\right\|_{L^{\gamma}(\Omega)} d \tau \\
& \leqslant C(\Delta t)^{-1 / \gamma}\left\|\left(T-T_{h}\right) u_{\beta}\right\|_{L^{\gamma}\left(t_{n}, t_{n+1}, L^{\gamma}(\Omega)\right)} .
\end{aligned}
$$


By Hölder's inequality, (2.24), (2.33), and (5.37), the second term on the right side of (5.35) may be bounded by

$$
\begin{aligned}
\left\|\left(T-T_{h}\right)\left(\partial^{+} u_{\beta}\right)^{n}\right\|_{L^{\gamma}(\Omega)}\left\|\left(u_{\beta}-U_{h}\right)^{n+1}\right\|_{L^{2+\nu}(\Omega)} \\
\leqslant C\left\|\left(T-T_{h}\right)\left(\partial^{+} u_{\beta}\right)^{n}\right\|_{L^{\gamma}(\Omega)}^{\gamma}+\frac{\eta}{4}\left\|\left(u_{\beta}-U_{h}\right)^{n+1}\right\|_{L^{2+\nu}(\Omega)}^{2+\nu} \\
\leqslant \frac{C}{\Delta t}\left\|\left(T-T_{h}\right) u_{\beta t}\right\|_{L^{\gamma}\left(t_{n}, t_{n+1}, L^{\gamma}(\Omega)\right)}^{\gamma} \\
+\frac{1}{4}\left(K_{\beta}\left(u_{\beta}\right)^{n+1}-K_{\beta}\left(U_{h}\right)^{n+1},\left(u_{\beta}-U_{h}\right)^{n+1}\right)
\end{aligned}
$$

where we hide the last term as above.

By (5.35)-(5.38),

$$
\begin{aligned}
& \frac{1}{2 \Delta t}\left\{\left\|\left(u_{\beta}-U_{h}\right)^{n+1}\right\|_{H_{h}^{-1}}^{2}-\left\|\left(u_{\beta}-U_{h}\right)^{n}\right\|_{H_{h}^{-1}}^{2}\right\} \\
& \quad+\frac{1}{2}\left(K_{\beta}\left(u_{\beta}\right)^{n+1}-K_{\beta}\left(U_{h}\right)^{n+1},\left(u_{\beta}-U_{h}\right)^{n+1}\right) \\
& \leqslant C(\Delta t)^{1 /(1+\nu)}\left\|T u_{\beta t t}\right\|_{L^{\gamma}\left(t_{n}, t_{n+1}, L^{\gamma}(\Omega)\right)}^{\gamma}+C(\Delta t)^{-1}\left\|\left(T-T_{h}\right) u_{\beta t}\right\|_{L^{\gamma}\left(t_{n}, t_{n+1}, L^{\gamma}(\Omega)\right)}^{\gamma} .
\end{aligned}
$$

Multiply by $\Delta t$ and sum on $n$ to obtain

$$
\begin{gathered}
\operatorname{Max}_{n}\left\|\left(u_{\beta}-U_{h}\right)^{n}\right\|_{H_{h}^{-1}}^{2}+\sum_{n}\left(K_{\beta}\left(u_{\beta}\right)^{n}-K_{\beta}\left(U_{h}\right)^{n},\left(u_{\beta}-U_{h}\right)^{n}\right) \cdot \Delta t \\
\leqslant C(\Delta t)^{\gamma}\left\|T u_{\beta t t}\right\|_{L^{\gamma}\left(0, T, L^{\gamma}(\Omega)\right)}^{\gamma}+C\left\|\left(T-T_{h}\right) u_{\beta t}\right\|_{L^{\gamma}\left(0, T, L^{\gamma}(\Omega)\right)}^{\gamma} .
\end{gathered}
$$

Use (5.27) and (5.40) to prove (5.30) with $m=\gamma$. Combine (2.33), (5.16), and (5.27) to verify (5.31) with $m=\gamma$.

When (3.47) is valid, we can establish bounds (5.30)-(5.33) with $m=2$. We replace (5.36) with the following bound for the second term in (5.35)

$$
\begin{aligned}
& \frac{1}{\Delta t} \int_{t_{n}}^{t_{n+1}} \int_{s}^{t_{n+1}}\left\|T u_{\beta t t}(\tau)\right\|_{H^{1}(\Omega)} d \tau d s \cdot\left\|\left(u_{\beta}-U_{h}\right)^{n+1}\right\|_{H^{-1}(\Omega)} \\
& \quad \leqslant C \cdot \sqrt{\Delta t} \cdot\left\|T u_{\beta t t}\right\|_{L^{2}\left(t_{n}, t_{n+1}, H^{1}(\Omega)\right)}\left\|\left(u_{\beta}-U_{h}\right)^{n+1}\right\|_{H^{-1}(\Omega)} \\
& \quad \leqslant C \cdot \Delta t \cdot\left\|T u_{\beta t t}\right\|_{L^{2}\left(t_{n}, t_{n+1}, H^{1}(\Omega)\right)}^{2}+C\left\|\left(u_{\beta}-U_{h}\right)^{n+1}\right\|_{H^{-1}(\Omega)}^{2} \\
& \quad \leqslant C \cdot \Delta t \cdot\left\|T u_{\beta t t}\right\|_{L^{2}\left(t_{n}, t_{n+1}, H^{1}(\Omega)\right)}^{2}+C\left\|\left(u_{\beta}-U_{h}\right)^{n+1}\right\|_{H^{-1}(\Omega)}^{2} \\
& \quad \leqslant C \cdot \Delta t \cdot\left\|\nabla K_{\beta}\left(u_{\beta}\right)_{t}\right\|_{L^{2}\left(t_{n}, t_{n+1}, L^{2}(\Omega)\right)}^{2}+C\left\|\left(u_{\beta}-U_{h}\right)^{n+1}\right\|_{H^{-1}(\Omega)}^{2},
\end{aligned}
$$

where we have used the identity

$$
-T u_{\beta t t}=K_{\beta}\left(u_{\beta}\right)_{t}-\frac{1}{|\Omega|} \int_{\Omega} K_{\beta}\left(u_{\beta}\right)_{t} d x
$$

and the consequent relation

$$
\left\|T u_{\beta t t}\right\|_{H^{\prime}(\Omega)}=\left\|\nabla K_{\beta}\left(u_{\beta}\right)_{t}\right\|_{L^{2}(\Omega)} .
$$


Next, use (2.24), (2.33), and (4.31) to see that

$$
\begin{aligned}
\left\|\left(u_{\beta}-U_{h}\right)^{n+1}\right\|_{H^{-1}(\Omega)}^{2} & \\
= & \left\|\left(u_{\beta}-U_{h}\right)^{n+1}\right\|_{H_{h}^{-1}}^{2}+\left(\left(T-T_{h}\right)\left(u_{\beta}-U_{h}\right)^{n+1},\left(u_{\beta}-U_{h}\right)^{n+1}\right) \\
\leqslant & \left\|\left(u_{\beta}-U_{h}\right)^{n+1}\right\|_{H_{h}^{-1}}^{2}+C h^{2}\left\|\left(u_{\beta}-U_{h}\right)^{n+1}\right\|^{2} \\
\leqslant & \left\|\left(u_{\beta}-U_{h}\right)^{n+1}\right\|_{H_{h}^{-1}}^{2}+C h^{2}\left\|\left(u_{\beta}-U_{h}\right)^{n+1}\right\|_{L^{2+\nu}(\Omega)}^{2} \\
\leqslant & \left\|\left(u_{\beta}-U_{h}\right)^{n+1}\right\|_{H_{h}^{-1}}^{2}+\frac{1}{4} \eta\left\|\left(u_{\beta}-U_{h}\right)^{n+1}\right\|_{L^{2+\nu}(\Omega)}^{2+\nu}+C h^{2(2+\nu) / \nu} \\
\leqslant & \left\|\left(u_{\beta}-U_{h}\right)^{n+1}\right\|_{H_{h}^{-1}}^{2}+\frac{1}{4}\left(K_{\beta}\left(u_{\beta}\right)^{n+1}-K_{\beta}\left(U_{h}\right)^{n+1}, u_{\beta}^{n+1}-U_{h}^{n+1}\right) \\
& +C h^{2 \gamma} .
\end{aligned}
$$

Combining (5.35), (5.38), (5.41), and (5.42) yields

$$
\begin{aligned}
& \frac{1}{2 \Delta t}\left\{\left\|\left(u_{\beta}-U_{h}\right)^{n+1}\right\|_{H_{h}^{-1}}^{2}-\left\|\left(u_{\beta}-U_{h}\right)^{n}\right\|_{H_{h}^{-1}}^{2}\right\} \\
& \quad+\frac{1}{2}\left(K_{\beta}\left(u_{\beta}\right)^{n+1}-K_{\beta}\left(U_{h}\right)^{n+1}, u_{\beta}^{n+1}-U_{h}^{n+1}\right) \\
& \leqslant C(\Delta t)\left\|\nabla K_{\beta}\left(u_{\beta}\right)\right\|_{t}^{2} \|_{L^{2}\left(t_{n}, t_{n+1}, L^{2}(\Omega)\right)}^{2} \\
&+C(\Delta t)^{-1}\left\|\left(T-T_{h}\right) u_{\beta t}\right\|_{L^{\gamma}\left(t_{n}, t_{n+1}, L^{\gamma}(\Omega)\right)}^{\gamma}+C\left\|\left(u_{\beta}-U_{h}\right)^{n+1}\right\|_{H_{h}^{-1}}^{2} .
\end{aligned}
$$

Multiply (5.43) by $2 \Delta t$ and sum on $n$ using the discrete Gronwall lemma

$$
\begin{gathered}
\operatorname{Max}_{n}\left\|\left(u_{\beta}-U_{h}\right)^{n}\right\|_{H_{h}^{-1}}^{2}+\sum\left(K_{\beta}\left(u_{\beta}\right)^{n}-K_{\beta}\left(U_{h}\right)^{n},\left(u_{\beta}-U_{h}\right)^{n}\right) \cdot \Delta t \\
\quad \leqslant C(\Delta t)^{2}\left\|\nabla K_{\beta}\left(u_{\beta}\right)_{t}\right\|_{L^{2}\left(0, T, L^{2}(\Omega)\right)}^{2}+C\left\|\left(T-T_{h}\right) u_{\beta t}\right\|_{L^{\gamma}\left(0, T, L^{\gamma}(\Omega)\right)}^{\gamma} .
\end{gathered}
$$

Use (3.47) and (5.44) to prove (5.30) with $m=2$. Use (2.33), (5.17), and (5.44) to prove (5.31) with $m=2$.

Under the quasiuniformity assumption (4.2), we have

(5.45) $\operatorname{Max}_{n}\left\|\left(u-P_{h} U_{h}\right)^{n}\right\|_{H^{-1}(\Omega)}$

$$
\begin{aligned}
& \leqslant \operatorname{Max}_{n}\left\{\left\|\left(u-u_{\beta}\right)^{n}\right\|_{H^{-1}(\Omega)}+\left\|\left(I-P_{h}\right) u_{\beta}^{n}\right\|_{H^{-1}(\Omega)}+\left\|P_{h}\left(u_{\beta}-U_{h}\right)^{n}\right\|_{H^{-1}(\Omega)}\right\} \\
& \leqslant C \operatorname{Max}_{n}\left\{\left\|\left(u-u_{\beta}\right)^{n}\right\|_{H^{-1}(\Omega)}+\left\|P_{h}\left(u_{\beta}-U_{h}\right)^{n}\right\|_{H_{h}^{-1}}\right\}+C h^{\gamma},
\end{aligned}
$$

where we have used (4.23), (4.25), and (4.38). Bounds (4.23), (4.25), (4.37), (4.38), (5.16)-(5.17), (5.40), (5.44), and (5.45) yield (5.33).

Under the hypotheses of Theorem 5.1, we may use (3.15), (3.26), (3.43), and (3.47) to see that

$$
\left\|u_{\beta t}\right\|_{L^{2}\left(L^{2}\right)}+\left\|\nabla K_{\beta}\left(u_{\beta}\right)_{t}\right\|_{L^{2}\left(L^{2}\right)} \leqslant C, \quad 0 \leqslant \beta \leqslant 1
$$


so that (4.31) and Lemma 5.6 imply (5.4)-(5.6). Combine (3.3), (3.55), (4.48), and Lemma 5.6 to verify Theorem 5.2. Use (3.3), (4.59), and Lemma 5.5 to prove Theorem 5.3. Finally, use (3.3), (4.31), (4.48), and Lemma 5.6 to justify the conclusions of Theorem 5.4 .

Remark. The argument of Lemma 4.6 may be used to show that $u_{\beta}$ can be replaced by $U_{h}$ in estimates (4.37) and (4.38). To see that bound (4.39) holds for $U_{h}$, just substitute $\partial V_{h} / \partial t \in M_{h}$ into Eq. (4.4), integrate in time, and recall that $V_{h}=$ $K_{\beta}\left(U_{h}\right)$.

This remark allows us to delete the projection $P_{h}$ preceding $U_{h}$ on the left sides of error estimates (4.8), (4.10), (4.12), (4.14), (4.16), (4.18), (4.43), (4.44), (5.5), (5.6), (5.11), (5.12), (5.14), (5.15), (5.32), and (5.33).

Acknowledgements. The author would like to thank his thesis advisor, Professor Jim Douglas, Jr., for his contributions to this work. Todd Dupont also made many helpful suggestions. The author would also like to thank Donald G. Aronson, Philippe Benilan, Michael G. Crandall, Hans G. Kaper, Richard P. Kendall, Gary K. Leaf and Mary F. Wheeler for their encouragement and advice. The author is grateful to Argonne National Laboratory for having sponsored this research and to the Exxon Production Research Company for the preparation of this manuscript.

Department of Mathematics

Tulane University

New Orleans, Louisiana 70118

1. D. G. AROnSON, “Regularity properties of flows through porous media," SIAM J. Appl. Math., v. 17. 1969, pp. 461-467.

2. D. G. ARONSON, "Regularity properties of flows through porous media: The interface," Arch. Rational Mech. Anal., v. 37. 1970, pp. 1-10.

3. D. G. Aronson, "Regularity properties of flows through porous media: A counterexample," SIAM J. Appl. Math., v. 19, 1970, pp. 299-307.

4. P. Benilan, Equations d'Evolution dans un Espace de Banach Quelconque et Applications, Doctoral Thesis, Orsay, 1972.

5. P. Benilan, Personal communication.

6. H. Brezis, Operateurs Maximaux Monotones et Semi-Groupes de Contractions dans les Espaces de Hilhert, American Elsevier, New York, and North-Holland, Amsterdam, 1973.

7. P. G. Ciarlet, The Finite Element Method for Elliptic Problems, American Elsevier, New York, and North-Holland, Amsterdam, 1978.

8. J. Douglas, JR., Flow of Fluids in Porous Media, Proc. Internat. Congr. Math., Vol. 3, 1970, pp. $65-69$.

9. J. Douglas, JR., T. Dupont \& L. Wahlbin, "Optimal $L^{\infty}$ error estimates for Galerkin approximations to solutions of two-point boundary value problems," Math. Comp., v. 29, 1975, pp. 475-483.

10. B. F. Knerr, “The porous medium equation in one dimension," Trans. Amer. Math. Soc. (To appear.)

11. O. Ladyzhenskaya, V. A. Solonnikov \& N. N. URal'Ceva, Linear and Quasilinear Equations of Parabolic Type, Transl. Math. Monographs, Vol. 23, Amer. Math. Soc., Providence, R. I., 1968.

12. J. L. Lions, Quelques Méthodes de Résolution des Problémes aux Limites non Linéaires, Dunod, Paris, 1969.

13. J. Nitsche, $L^{\infty}$ Convergence of Finite Element Approximations, Proc. Second Conf. on Finite Elements, Rennes, France, 1975.

14. J. Nitsche, On $L^{\infty}$ Convergence of the Finite Element Approximations to the Solution of a Nonlinear Boundary Value Problem, Conf. on Numerical Analysis, Dublin, Ireland, 1976.

15. O. A. Oleinik, On Some Degenerate Quasilinear Parabolic Equations, Seminari dell'Instituto Nazionole di Alta Mathematica, 1962-1963. 
16. O. A. Oleinik, A. S. Kalashnikov \& Czou Yui-Lin, "The Cauchy problem and boundary problems for equations of the type of nonstationary filtration," Izv. Akad. Nauk. SSSR Ser. Mat., v. 22, 1958, pp. 667-704. (Russian)

17. D. W. Peaceman, Fundamentals of Numerical Reservoir Simulation, American Elsevier, New York, and North-Holland, Amsterdam, 1977.

18. M. E. Rose, Numerical Methods for a General Class of Porous Medium Equations, Argonne National Laboratory Report, Argonne, Illinois, 1979.

19. M. E. RosE, “Numerical methods for flows through porous media-II," Comput. Math. Appl., v. 20, 1980, pp. 99-122.

20. R. Scort, "Optimal $L^{\infty}$-estimates for the finite element method on irregular meshes," Math. Comp., v. 30, 1976, pp. 681-697.

21. E. Stein \& G. Weiss, Introduction to Fourier Analysis on Euclidean Spaces, Princeton Univ. Press, Princeton, N. J., 1971.

22. S. Sternberg, Lectures on Differential Geometry, Prentice-Hall, Englewood Cliffs, N. J., 1964.

23. M. F. WHEeler, 'A priori $L^{2}$ error estimates for Galerkin approximations to parabolic partial differential equations," SIAM J. Numer. Anal., v. 10, 1973, pp. 723-759. 